\title{
LES of synthetic jets in boundary layer with laminar separation caused by adverse pressure gradient
}

\author{
Tetsuya Ozawa, Samuel Lesbros and Guang Hong* \\ University of Technology, Sydney (UTS), Australia
}

*Corresponding author. Mail address: Faculty of Engineering, University of Technology Sydney, PO Box 123, Broadway, NSW 2007, Australia.

Email: guang.hong@uts.edu.au, Telephone: +61 29514 2678, Fax: +61 295142655

\begin{abstract}
In the development of synthetic jet actuators (SJAs) for active flow control, numerical simulation has played an important role. In controlling the boundary layer flow separation, an integrated numerical model which includes both the baseline flow and the SJA is still in its initial stage of development. This paper reports preliminary results of simulating the interaction between a synthetic jet and a laminar separation bubble caused by adverse pressure gradient in a boundary layer. The computational domain was three-dimensional and Large-eddy simulation (LES) was adopted. The initial and boundary conditions were defined using or referring to our wind tunnel experimental results. Prior to numerically simulating the interaction between the synthetic jets and the baseline flow, a numerical model for simulating the separation bubble was developed and verified. In the numerical model including the SJA, the synthetic jet velocity at the exit of the SJA was defined as an input. The numerical model was further verified by comparing the simulation with experimental results. Based on reasonable agreement between the numerical and experimental results, simulations were carried out to investigate the dependency of flow control using synthetic jets on the forcing frequency, focused on the lower frequency range of the Tollmien-Schlichting (T-S) instability, and on the forcing amplitude which was represented by the maximum jet velocity at the exit of the SJA. Supporting the hypothesis based on the
\end{abstract}


experiment, LES results showed that the forcing frequency had stronger influence on SJA's effective elimination of the separation bubble than the forcing amplitude did.

Key words: synthetic jet actuator, micro sensor, numerical modeling, flow control.

\section{Nomenclature}

$c_{P}-$ pressure coefficient

$C_{s}-$ Smagorinsky model constant

$d_{0}$ - diameter of the orifice of the SJA

$f-\mathrm{SJA}$ 's forcing frequency

$\bar{f}$ - filtered value

$F^{+}$- non-dimensional frequency

$G-$ filter function

$L_{x}, L_{y}$ and $L_{z}$ - dimension of the computational domains in the Cartesian directions $x, y$ and $z$ $N$ - sample size of experimental data

$P(x)$ - pressure distribution in the streamwise direction

$\bar{P}$ - pressure parameter

$\bar{S}_{i j}$ - strain tensor of grid-filtered velocity

$t$ - time

$u_{i}-$ velocity in each of the three dimensions in Navier-Stokes equation, or the $i t h$ sample data of the instantaneous streamwise velocity in Eq. (11)

$\bar{u}$ - sample mean of the streamwise velocity

$u_{a v g}=0-$ zero streamwise velocity at an averaged inflectional point

$u$ ' - streamwise velocity fluctuation

$u_{\max }-$ maximum velocity fluctuation in the fluctuating velocity profile 
$\hat{u}_{i}, \hat{u}_{j}$ - sub-grid scale velocity

$U$ - free stream velocity

$u_{i}-$ grid-filtered velocity

$U_{\text {jet }}-$ jet velocity component in streamwise direction at the exit of SJA

$V_{\text {jet }}-$ jet velocity component in direction normal to the wall at the exit of SJA

$W_{\text {jet }}-$ jet velocity component in the spanwise direction at the exit of SJA

$x-$ streamwise direction in the computational domain. $x=0$ at the exit of the SJA.

$x_{i}, x_{j}-$ space coordinates in Navier-Stokes equation

$v$ - kinematic viscosity of the working fluid

$v_{\mathrm{e}}$ - eddy viscosity

$y$ - normal to wall direction in the computational domain

$z$ - spanwise direction in the computational domain

$\rho$ - density of the working fluid

$\tau_{i j}-$ sub-grid scale stress

$\delta$ - boundary layer thickness

$\delta^{*}$ - displacement thickness

$\Delta-$ width of the filter

$\theta$ - momentum thickness

\section{Introduction}

Despite many 'unknowns' in modeling the fluid flows, the challenge of CFD is now compounded with the introduction of active flow control technologies [[28]]. In developing synthetic jet actuators (SJAs) for active flow control, extensive work has been conducted on numerical modeling of the synthetic jet generated in a quiescent external flow condition (in the 
absence of a cross flow) [[11],[14],[17],[25]]. In this numerical modeling, the neighborhood of the jet exit was simulated. Kral et al [[11]] aimed to model the boundary condition at the exit of the orifice of the SJA. They did not model the air flow inside the cavity but examined three different velocity distributions along the axial centerline of the orifice. They also investigated various jets, laminar and turbulent, pulsed and steady, and achieved very good agreement between the numerical simulation and the experimental measurement. The models in $[[14],[17],[25]]$ included the flow behavior in the cavity of the actuator and simulated not only the jets generated but also the compression/intake and expansion/discharging processes in the cavity.

Most of the reported work on numerical simulation of synthetic jets has been focused on the jets in a quiescent condition. Few publications have reported numerical simulation of the interaction between the synthetic jets and the baseline flow to be controlled. Mittal et al simulated the synthetic jet at the exit of the SJA with cross flow in a boundary layer under a zero pressure gradient [[17]]. Allan et al [[2]] investigated the numerical simulation of a 2-D airfoil controlled by synthetic jets. They demonstrated the CFD model coupled with the model for rigid body motion. Parekh et al [[20]] numerically simulated the experiments of Honohan et al [[10]] that studied separation control on a thick airfoil using synthetic jet action. Their model successfully predicted the reattachment dynamics and the dependence of controlling reattachment on forcing frequency.

Different flow models and numerical approaches have been consistent in development and enhancement through their applications. The turbulence models and solvers used in simulating the synthetic jets in a quiescent condition include a two-dimensional incompressible flow model with Reynolds-averaged Navier-Stokes (RANS) [[11]] or Direct Numerical Simulation (DNS) 
[[17]], and three-dimensional model with DNS [[21]]. In the 2-D simulation of separation control over an airfoil using synthetic jets, unsteady RANS [[2]] and hybrid RANS/LES derived from combining the best features of RANS and LES [[20]] have been used.

Prior to numerically modeling the synthetic jets interacting with the baseline flow to be controlled, it is necessary to have a numerical model ready for the baseline flow such as a laminar separation bubble caused by adverse pressure gradient in the boundary layer. The difficulties in modeling the separation bubble, especially the transition from laminar to turbulence, have been well known. As commented by Gad-el-Hak [[3]], current inaccuracies in turbulence modeling can severely degrade CFD predictions once separation has occurred. The over-prediction of the production of turbulence kinetic energy and dissipation were reported in [[22]]. However, while he pointed out the problems, Gad-de-hak also justified that the essence of separation control was the calculation of attached flows, estimation of separation location, and indeed whether or not separation would occur [[3]]. This has given us certain confidence in using CFD as an alternative tool for developing synthetic jet actuators and also raised our cautiousness for using CFD properly.

A closed region formed by laminar separation followed by turbulent attachment is known as a laminar separation bubble. Laminar separation caused by adverse pressure gradient is a common phenomenon in aerodynamic devices. Alam and Sandham reviewed the numerical simulation of laminar separation bubbles from the first attempt in 1990 to 1998 [[1]], including LES used by Wilson and Pauley to simulate two-dimensional large structures [[31]]. They commented that only a few simulations provided good solution of the reattaching and developing turbulent boundary layer. 
Direct numerical simulation (DNS) of the incompressible Navier-Stokes equations was applied to simulating the 'short' laminar separation bubble caused by adverse pressure gradient in a boundary layer [[1],[23],[32]]. Alam and Sandham carried out simulations in both two- and three-dimensions, and concluded that two-dimensional simulation failed to capture many of the detailed features achieved from the three-dimensional simulation. They compared their simulation results of transition length-based Reynolds number varying with local turbulence level with the experimental ones, and showed good agreements. Rist used his DNS results to give an introduction and an overview on instability and transition mechanisms in laminar separation bubbles [[23]]. Regarding the numerical methods, Rist commented that RANS did not cover the unsteady flow physics and LES was not yet well proven for transitional flow.

As reviewed above, although the numerical simulations of a synthetic jet in the absence of a cross flow and of a separating boundary layer have been performed respectively, an integrated numerical model including the synthetic jets in a separating boundary layer, is still being developed. Rumsey summarized in 2008 that there had not been many published results for computation of synthetic jet in a cross flow since the CFD workshop organized by NASA in 2004 [26]. Therefore, Work reported in this paper aimed to develop a three-dimensional LES model which was reasonably capable of simulating the laminar separation bubble caused by adverse pressure gradient, to provide a numerical baseline flow required for predicting the effectiveness of a synthetic jet actuator (SJA) in controlling boundary layer flow separation.

\section{Synthetic jet actuator}

A synthetic jet, originated from the idea of acoustic streaming [[15]], has been known to have zero-net-mass but non-zero momentum fluid flux generated by a device such as a piezooscillator. It has emerged as a versatile actuator with potential applications ranging from 
separation and turbulence control to thrust vectoring, and augmentation of heat transfer and mixing [[3],[8],[12],[16],[30]]. A unique feature of synthetic jets is that they are formed entirely from the working fluid of baseline flow without requirement of additional mass supply [[6]]. As shown in the schematic in Fig. 1(a), a synthetic jet actuator consists of a cavity and an oscillating diaphragm. The jet is synthesized by oscillatory flow through a small orifice to a cavity. The flow is induced by a vibrating diaphragm which forms the bottom wall of the cavity.
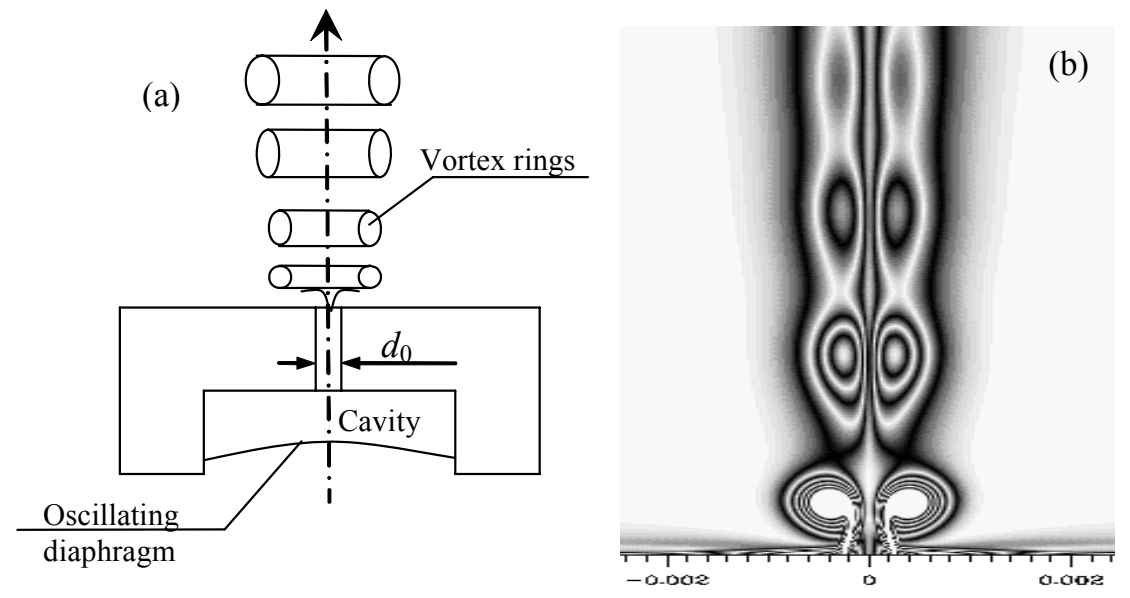

Figure 1 (a) Schematic diagram of a synthetic jet actuator

(b) Instantaneous vortex contours of LES simulation of a synthetic jet in a quiescent condition

The synthetic jet actuator (SJA) in the present study used piezoelectric material for driving the oscillating diaphragm, as it promotes desirable characteristics such as low power consumption, fast response, reliability, and low cost [[6]]. As illustrated in Fig. 1(a), the SJA has an orifice in the face opposite the membrane. This orifice is open to the boundary layer flow and has a diameter, $d_{0}$, of $0.5 \mathrm{~mm}$. The actuator membrane is a thin circular brass disc, $0.25 \mathrm{~mm}$ in thickness, held firmly at its perimeter. A piezoceramic disc is bonded to the outside face of the membrane. As specified by the manufacturer, the lowest resonant frequency of the membrane is $900 \mathrm{~Hz}$ and its lump sum capacitance is approximately $140 \mathrm{nF}$. In the wind tunnel experiments, as shown in Fig. 1, the SJA was installed underneath a flat plate over which the streamwise velocity was measured in a boundary layer developing under an adverse pressure gradient. In 
operation, the SJA was driven by a sine wave signal generated by a standard electrical function generator. An air jet was synthesized by oscillatory flow in and out of the cavity through the orifice open to the boundary layer. As many other actuators for flow control, SJA tends to be a 2D excitation device. In Fig. 1(b) are the vortex contours from a 2-D LES simulation of the synthetic jet in a quiescent condition. It shows the formation and development of the 2-D vortex rings of the jet without cross-flow.

\section{Experimental Background}

The experiments investigating the synthetic jet actuator for controlling laminar separation were performed in the low speed wind tunnel in the Aerodynamics Laboratory at the University of Technology, Sydney. As shown in Fig. 2, in the working section, a fairing was set above an aluminum flat plate with its angle adjustable for establishing the desired pressure gradient, similar to that of a diffusion compressor blade. The flat plate, located $1200 \mathrm{~mm}$ from the working section entrance, has a high quality surface finish. The leading edge of the upper surface is of slender elliptical form and the plate has a $0.25^{\circ}$ negative incidence to avoid leading edge separation. Static taps were located every $25 \mathrm{~mm}$ along the streamwise centerline of the flat plate for pressure measurement using a multi-tube manometer. The SJA was installed underneath the flat plate and on the streamwise centerline. The exit of the SJA was an orifice open to the boundary layer flow. The axial center of the orifice of the SJA was located $305 \mathrm{~mm}$ to the leading edge. The streamwise velocity was measured using a Dantec hot wire anemometry in the boundary layer flow over the upper surface of the flat plate. The 'Streamline' box in Fig. 2 shows a package of software and hardware for interfacing between the computer and the hotwire probe and for data acquisition. The probe was traversed in streamwise $(x)$ and normal $(y)$ directions. The sample rate was $6 \mathrm{kHz}$ and the sample size of each realization was 4096 . 
Measurements were made at $x=40 \sim 160 \mathrm{~mm}$ downstream of the SJA $(345-465 \mathrm{~mm}$ from the leading edge) at $20 \mathrm{~mm}$ intervals. At each of the $x$ stations, the hot wire probe was positioned at $y$ $=0.2 \mathrm{~mm}$ to beyond the edge of the boundary layer. At each measurement position, the streamwise velocity was recorded in both conditions of jet on and off. The Reynolds number in the measurement region, $x=40 \sim 160 \mathrm{~mm}$, was in a range of $1.78 \times 10^{5} \sim 2.24 \times 10^{5}$. The forcing voltage for the synthetic jets was $\pm 7.5 \mathrm{~V}$, and forcing frequency $100 \mathrm{~Hz}$. The freestream velocity was $8.0 \mathrm{~m} / \mathrm{s}$ and freestream turbulence was $0.5 \%$ measured at $x=-50 \mathrm{~mm}$ which was the position with the minimum pressure. More details about the wind tunnel tests are provided in $[8,9]$.

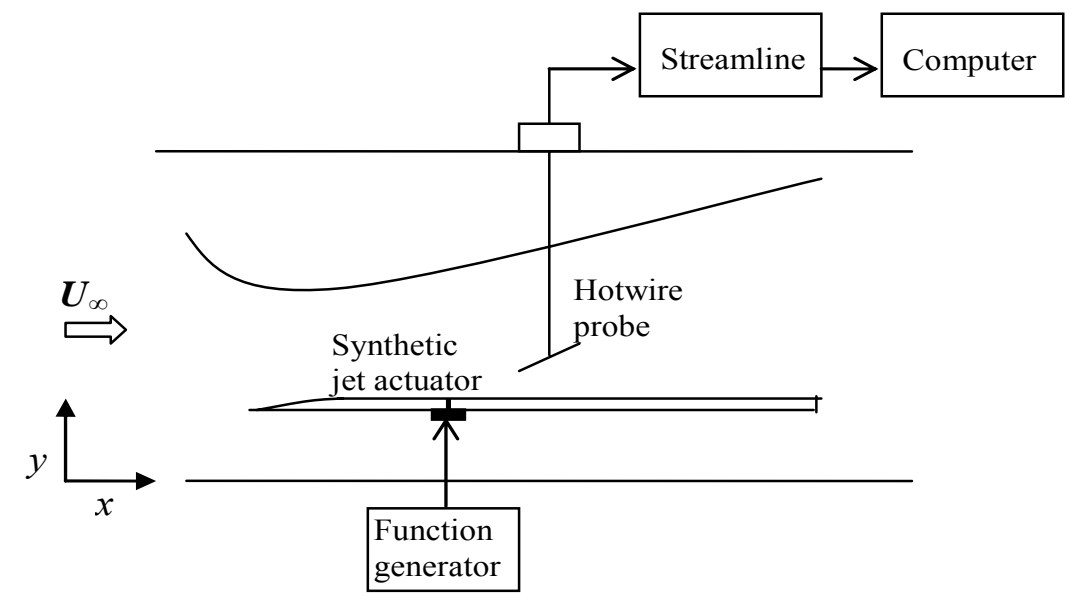

Figure 2 Side view of the experimental setting in the working section of the wind tunnel

\section{Numerical Simulation}

\subsection{Computational domain}

Figure 3 shows the computational domain. $x$ corresponds to the streamwise direction, $y$ the wall-normal direction and $z$ the spanwise direction. The bottom of the computational domain is the upper surface of the flat plate in Fig. 2. To facilitate comparison with the experiments, the dimensional length units are used. The dimensions of the computational domain are $L_{x}=200 \mathrm{~mm}$, $L_{y}=60 \mathrm{~mm}$ and $L_{z}=90 \mathrm{~mm}$ in streamwise, wall-normal and spanwise directions respectively. 
The dimension in $y$ direction was empirically set to be sufficiently high so that the velocity field at the top of the domain was not to be influenced by the separation in the boundary layer at the bottom of the computational domain. The domain is symmetric about the streamwise centerline at $z=0$. The reference position, $x=0, y=0$ and $z=0$, is the axial centre of the orifice at the exit of the SJA. The inlet of the computational domain is $20 \mathrm{~mm}$ upstream of the exit of the SJA, defined as $x=-20 \mathrm{~mm}$. In the course of developing this numerical simulation, it was noticed that the shape of the SJA's orifice, round or square, had insignificant effect on the simulation outputs. Therefore, the round geometry of the orifice was replaced by a simple squared geometry in simulation.

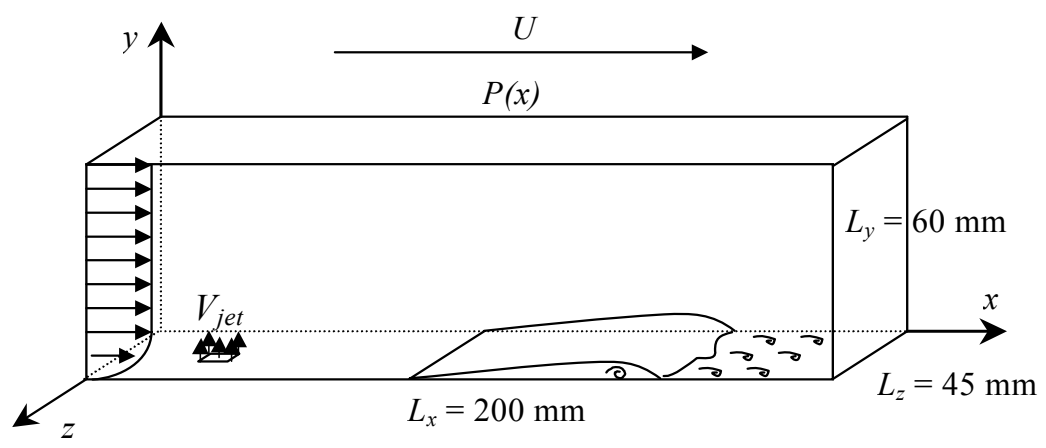

Figure 3 Schematic of computational domain

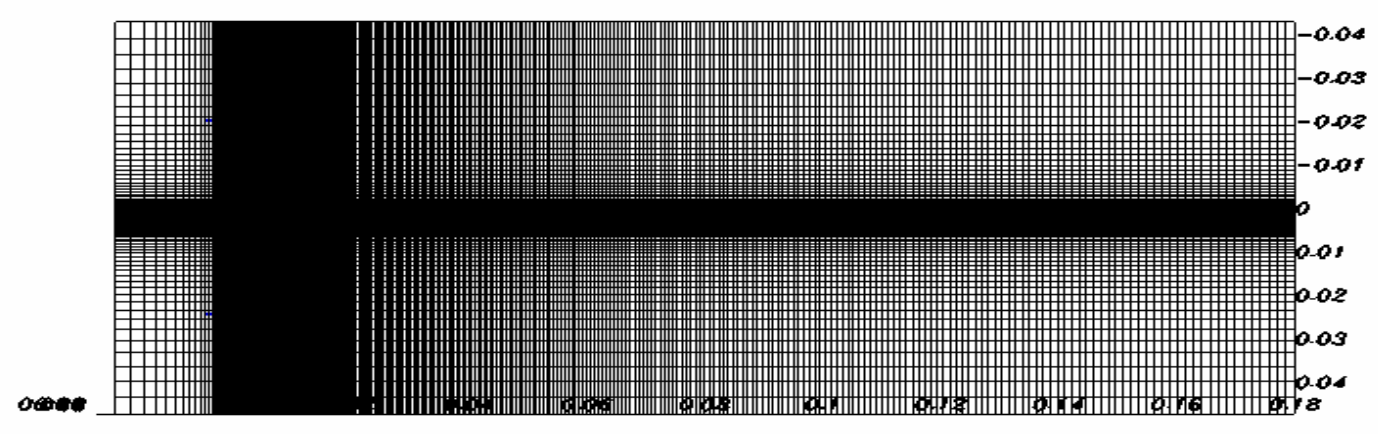

(a) 


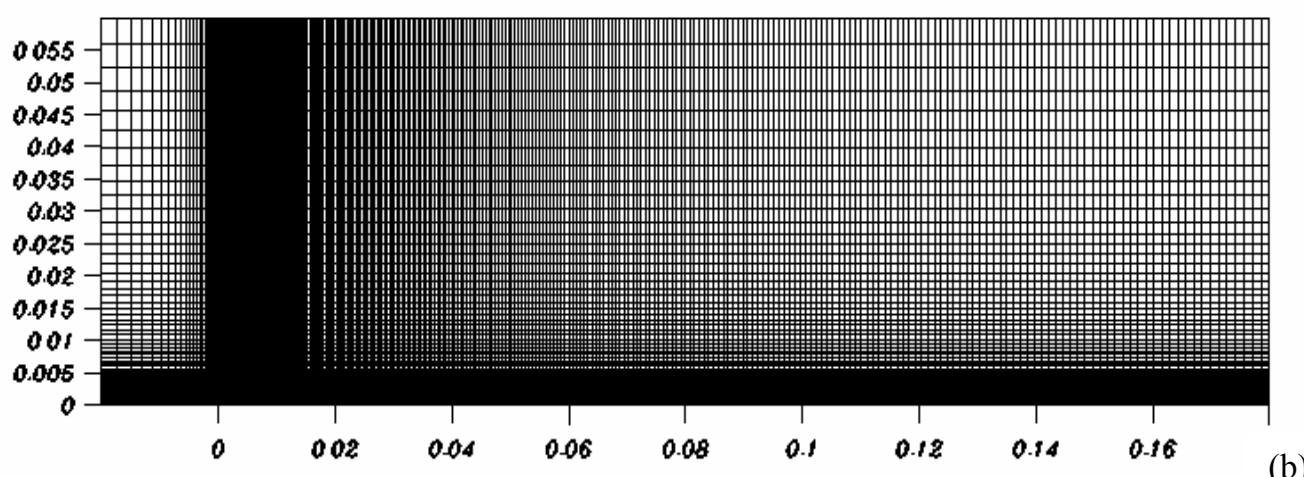

Figure 4 Mesh structure in the computational domain, (a) top view and (b) side view. The unit of the coordinates is meter.

The computational field was decomposed into six sections which were solved in six parallel processors in a cluster system. This decomposition was made to support the grid size and to reduce the computational time. As the project aimed to simulate the boundary layer interacting with a synthetic jet, the mesh in the boundary layer and in the adjacent area of the orifice of the SJA was finer. As shown in Fig. 4, the mesh gradually became coarse with the distance further from the exit of the SJA. In accordance with LES standard, grid spacing near the orifice was determined based on the diameter of the orifice $d_{0}$ to be $d_{0} / \Delta x=10, d_{0} / \Delta y=30, d_{0} / \Delta z=10$. The reducing factor was 0.5 . The number of grids in wall-normal direction in the boundary layer was 40, sufficient for LES [[27]]. Table 1 summarizes the dimensions of the computational domain and the grid distribution.

Table 1 Dimensions of the computational domain and grid distribution

\begin{tabular}{cccc}
$L_{x}(\mathrm{~mm})$ & $L_{y}(\mathrm{~mm})$ & $L_{z}(\mathrm{~mm})$ & Grid points \\
300 & 60 & 90 & $312 \times 82 \times 105$ \\
\hline \hline
\end{tabular}

\subsection{Large eddy simulation}

Large eddy simulation (LES) was adopted in this numerical simulation and a commercial code, CFD-ACE, was used as a solver. The governing equations for this flow are the equations of the 
incompressible Navier-Stokes and the continuity equations. In LES, the velocity components in the Navier-Stokes equations are decomposed into grid scale and sub-grid scale velocity, $U_{i}+\hat{u}_{i}$, through the filter in space. Operationally, the filter is described as

$$
\bar{f}(x, t)=\frac{1}{2 T} \int_{-T}^{T} f\left(x^{\prime}, t\right) d t G\left(x-x^{\prime}, t, \Delta_{f}\right) d x^{\prime}
$$

Where $\bar{f}$ represents the filtered value of the field variable, $G$ denotes the filter which is a top hat filter function. $\Delta_{f}$ is the filter width. The filtered Navier-Stokes and continuity equations become as follows.

$$
\begin{aligned}
& \frac{\partial \overline{u_{i}}}{\partial x_{i}}=0 \\
& \frac{\bar{D} u_{i}}{D t}=\frac{\partial}{\partial x_{j}}\left[v \frac{\partial \overline{u_{i}}}{\partial x_{j}}\right]-\frac{1}{\rho} \frac{\partial p}{\partial x_{i}}-\frac{\partial \tau_{i j}}{\partial x_{j}}
\end{aligned}
$$

Where $\tau_{i j}=\overline{\hat{u}_{i} \hat{u}_{j}}$ is the sub-grid scale (SGS) stress. Proposed methods for the closure of this term are mainly categorized into two groups-eddy viscosity models and scale similarity models. CFDACE provides the SGS Eddy Viscosity Coefficient model in which the SGS stress term is defined as:

$$
\begin{gathered}
\tau_{i j} \approx-v_{e} \bar{S}_{i j} \\
\bar{S}_{i j}=\frac{1}{2}\left(\frac{\partial \overline{u_{i}}}{\partial x_{j}}+\frac{\partial \overline{u_{j}}}{\partial x_{i}}\right)
\end{gathered}
$$

Where $v_{e}$ is the SGS eddy viscosity and $\overline{S_{i j}}$ is the strain tensor of grid-filtered velocity. Smagorinsky model based on isotropy-of-the-small-scales assumption has been broadly used due to its simplicity and accuracy [[29]]. Although the simulation of a homogeneous isotropic turbulence using Smagorinsky model may agree well with the experiment, SGS model does not 
have the versatility for various flow fields including the one in the present study. Thus, the Dynamic SGS model [[13]] was considered.

\subsection{Initial and boundary conditions}

The mean freestream velocity components at the inlet of the computational domain were defined as $U=9.1 \mathrm{~m} / \mathrm{s}$ (x-direction), $V=0 \mathrm{~m} / \mathrm{s}$ ( $y$-direction) and $W=0 \mathrm{~m} / \mathrm{s}$ (z-direction). Since the velocity profile at the inlet of the computational domain was not measured in the experiments, the numerical modelling of the inlet flow was required. A Blasius velocity profile of a laminar boundary layer was assumed at the inlet of the computational domain. The free stream mean velocity at the inlet was adjusted by the boundary layer thickness based Reynolds number $R e_{\delta}$, of approximately 500 . Artificial-minute disturbance at the inlet was adopted by generating Gaussian Random numbers. The root-mean-square (RMS) of the disturbance was $1 \%$ of the free stream velocity at the inlet. It was determined based on justifying the positions of the separation and reattachment points and the bubble length [[19]].

In the work reported in [[18]], convective boundary condition was used at the exit of the computational domain. An artificial 'buffer' zone was employed at the end of the computational domain to return the turbulent outflow to the Blasius laminar inflow profile, in order to apply periodic boundary conditions in the streamwise direction in the use of a fully spectral method. In our work, the outlet boundary condition of the computational domain was defined by the pressure value measured at the streamwise position of $x=180 \mathrm{~mm}$ in the wind tunnel test.

The condition at the top boundary of the computational domain in [[18]] was defined by a suction (normal) velocity profile $V_{t o p}(x)$ with a Gaussian distribution. By doing so, the separation and the reattachment points were fixed. Alternatively the entire wind tunnel was included in the model [[32]]. However, fixing the positions of the separation and reattachment 
does not suit the present study, as effective synthetic jets modify the separation and reattachment points. Instead of the velocity profile defined at the upper boundary of the domain, a pressure profile was applied with the following function.

$$
P\left(x, L_{y}, z, t\right)=P_{t o p}(x)
$$

Where $P$ is the static pressure as a function of the location and time. The setting of the models' height (in wall-normal direction) in this simulation was extensively examined and carefully decided [[19]]. The pressure coefficient from the experimental measurement, as shown in Fig. 5, was imposed onto the top boundary of the computational domain. In doing so, the free stream velocity and the adverse pressure gradient resulting in the boundary layer separation were defined in the LES model.

The non-slip boundary condition was set on the bottom wall of the computational domain with zero velocity defined at the wall $y=0$. The flow at the side walls of the computational domain was assumed to be symmetric about $z=0$. Applicable to both sides of the center, the symmetric boundary conditions generate a flow of period length $2 \times L_{z}$.

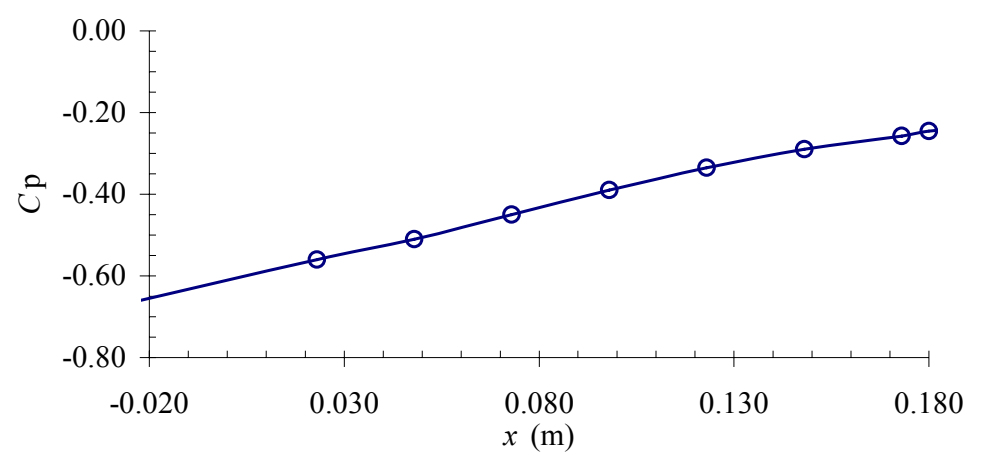

Figure 5 Static pressure distribution in the downstream direction

\subsection{Numerical Method}


By filtering the Navier-Stokes and the continuity equations in space, grid-filtered governing equation and Sub-grid scale stress (SGS) terms were produced. Dynamic Smagorinsky model was used for the approximation of SGS stress terms. Filtered Equations (2) and (3) were discretised in space using a hybrid scheme of the second-order central difference and first order upwind difference. 2,686,320 grid points were used, consisting of the concentrated mesh near the wall and the orifice of the SJA. $\Delta Y^{+}$at the first node off the wall in the boundary layer was less than 0.6 , and the corresponding $\Delta X^{+}$was less than 19 and $\Delta Z^{+}$less than 50. To test the grid independence, the mesh was refined with decreased $\Delta X^{+}$and $\Delta Z^{+}$. The characteristic parameters as the output showed insignificant difference with the finer mesh.

The forward and backward Euler and the second-order Crank-Nicolson methods were employed for time integration. The time step was set as $\Delta t=0.0002 \mathrm{~s}$ and the total number of time steps was 1600 in each run. The time step was set based on the forcing frequency of the SJA and the sample rate for data acquisition, $6 \mathrm{kHz}$, in the experiments. The simulation began at time $t=0$, with an analytical approximation to the steady-state solution. The flow was considered as transient and the residual convergence was less than $10^{-3}$. The CPU time for one simulation was about 36 hours for 500 time steps.

\section{Simulation of Laminar Separation Bubble}

In the development of the baseline flow model, a laminar separation bubble was formed in the boundary layer. Sample results are presented in the following sections to identify the separation bubble and to analyze the mechanics associated.

\subsection{Laminar Separation-Short Bubble}


Flow separation can be transitional separation or laminar separation, depending on the flow mode before the separation occurs. In order to identify the separated-flow transition modes, Hatman and Wang developed a prediction model for distinguishing three separated-flow transition modes, transitional separation, laminar separation-short bubble and laminar separationlong bubble [387]. The transitional separation has the onset of the transition occurring upstream of the separation point, and the other two have the onset of the transition downstream of the separation point by inflectional instability. In separated boundary layers, the model proposed by Hatman and Wang for separated-flow transition is based on the assumption that the transition to turbulence is a result of the superposition of the effects of two different types of instability: Kelvin-Helmholtz (K-H) instability and Tollmien-Schlichting (T-S) instability. The predominance of one type of instability determines the modes of separated-flow transition.

Laminar separation-short bubble was the one identified in our experimental studies [[8]] and simulated in our numerical studies [[19]]. It distinguishes itself from the transitional separation and the laminar separation-long bubble by a quick completion of transition. The laminar separation-short bubble mode occurs at moderate Reynolds numbers and mild adverse pressure gradients. The onset of the transition is induced downstream of the separation point by inflectional instability at a location coincidental with that of the maximum displacement in the shear layer. It is characterized by a quick transition completion due to a complex interaction between the separated shear layer and the reverse flow vortex. After the laminar shear layer detaches, the K-H instability sets in. The onset of the transition from laminar to turbulence should be situated close to the location of the maximum bubble elevation [[7]]. Then a short early transition region takes place. This early transition is shortened by a periodic ejection of turbulent fluid from the recirculating region into the detached shear layer. Instability waves of 
the T-S type, initiated upstream of the separation point, may still be present within the detached shear layer. The coalescence into turbulence takes place within the reattaching boundary layer, resulting in a short late transition region.

\subsection{Visualization of the separation bubble}

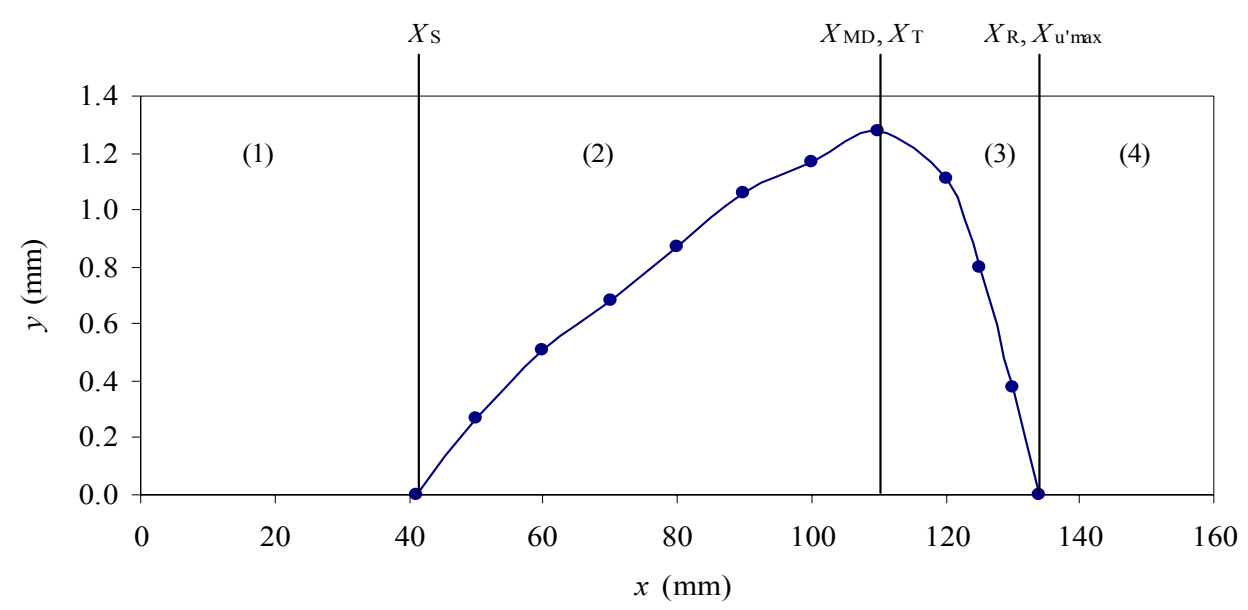

Figure 6 Laminar separation-short bubble viewed by positions with $u_{\text {avg }}=0$, on $z=0$ plane

Figure 6 shows the laminar separation-short bubble on the streamwise centerline from the LES simulation. The 'edge' of this separation bubble is viewed by the averaged positions with zero streamwise velocity, $u_{\mathrm{avg}}=0$, which exists in an inflectional velocity profile. Here $X_{\mathrm{S}}$ is the streamwise position of the separation point. $X_{\mathrm{MD}}$ is the streamwise position with the maximum displacement. $X_{\mathrm{T}}$ is the onset of the transition from laminar to turbulence, $X_{\mathrm{R}}$ is the streamwise position of the reattachment and $X_{\mathrm{u}}{ }^{\prime} \max$ is the streamwise position with the maximum fluctuating velocity. The separation $\left(X_{\mathrm{S}}\right)$ occurs at about $x=41 \mathrm{~mm}$ and the flow reattaches to the wall $\left(X_{\mathrm{R}}\right)$ at about $x=134 \mathrm{~mm}$. The transition onset $\left(X_{\mathrm{T}}\right)$ occurs at about $x=110 \mathrm{~mm}$. In region (1) is attached laminar flow. In region (2) is laminar shear layer detached from the wall. The streamwise length of region (2) is $77 \%$ of the bubble length. For a laminar separation-short bubble, the onset of the transition from laminar to turbulence, $X_{\mathrm{T}}$, occurs at a position 
coincidently with the maximum displacement $\left(X_{\mathrm{MD}}\right)$ [7]. The transition from laminar to turbulent is characterized by a short early transition region which is region (3) between $\left[X_{\mathrm{MD}}, X_{\mathrm{T}}\right]$ and $\left[X_{\mathrm{R}}\right.$, $\left.X_{\mathrm{u}^{\prime} \max }\right]$. The length of region (3) is $23 \%$ of the bubble length. In region (3), the mid-transition point $\left(X_{\mathrm{u}^{\prime} \max }\right)$ is merged with the reattachment point, $X_{\mathrm{R}}$. In region (4) is the developing turbulent flow after reattachment.

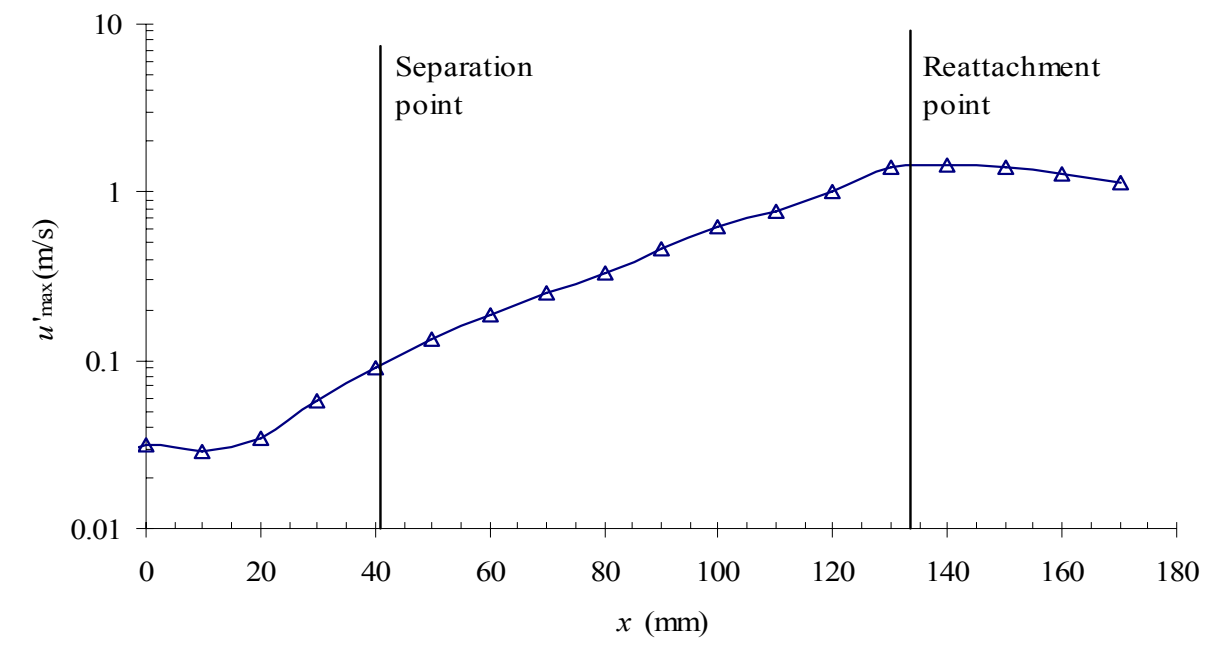

Figure 7 Variation of the maximum fluctuating velocity $u_{\text {max }}^{\prime}$ on streamwise centerline

For a laminar separation bubble in a short mode, caused by relatively moderate adverse pressure gradient, the vigorous mixing in the region of the maximum fluctuating velocity, $u_{\max }^{\prime}$, leads to reattachment [7]. The development of the 'maximum fluctuating velocity' along the streamwise centerline is shown in Fig. 7. Note that this 'maximum fluctuating velocity', $u$ ' max, is the maximum in the fluctuating velocity profile at a streamwise position. As shown in Fig. 7, $u_{\text {max }}^{\prime}$ remains constant up to the position about $20 \mathrm{~mm}$ upstream of the separation point. Inside the separation zone, $u^{\prime}{ }_{\max }$ increases exponentially until it reaches the position close to the reattachment point. The peak value of $u^{\prime}{ }_{\max }$ occurs at the reattachment point about $x=134 \mathrm{~mm}$. This shows one of the characteristics of a laminar separation-short bubble that the turbulence level is maximum at the reattachment, as reported in [738]. This result is also consistent with that 
of the DNS of a laminar separation bubble in [[24]]. The cause of this rapid increase can be explained as that the disturbance at the inlet is amplified by the Kelvin-Helmhortz $(\mathrm{K}-\mathrm{H})$ instability in the free shear layer flow. After the reattachment point where the peak value of $u^{\prime}{ }_{\max }$ is reached, the flow quickly becomes turbulent, as shown by the decrease of the maximum fluctuating velocity in Fig. 7.

\subsection{Transition from laminar to turbulent}

Simulation results in Figures 6 and 7 show that the laminar separation bubble, including the separation point and the re-attachment, was well captured. However, questions remain for the capability of this LES model to simulate the transition from laminar to turbulent. Vorticity may be used to identify the mixing between the sub-layer and the rest of the boundary layer. Figure 8 presents the iso-surface of the instantaneous vorticity of 2000 near the wall. Note that the distance is in the unit of meter and the spectrum bar gives the scale of the time averaged streamwise velocity. As shown in Fig. 8, the mixture starts to be enhanced at about $x=110 \mathrm{~mm}$, where the flow is still separated. Following this, the re-attachment occurs very quickly. It can also be observed that the large-scale waves transfer in streamwise direction and the laminar layer breaks down into streak structure downstream, similar phenomenon as described in [[1]]. After the reattachment occurs at about $x=134 \mathrm{~mm}$, the flow structure becomes finer and more complicated. Further downstream, the turbulence level increases quickly. 


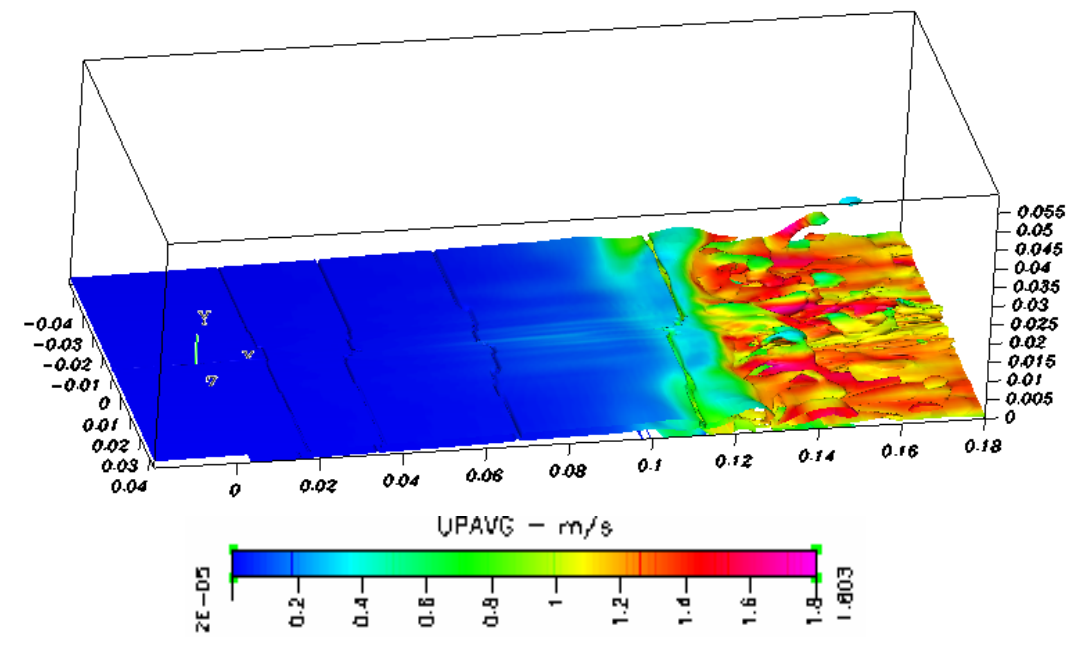

Figure 8 Iso-surface of instantaneous vorticity of 2000 (The spectrum bar shows the scale of the mean streamwise velocity)

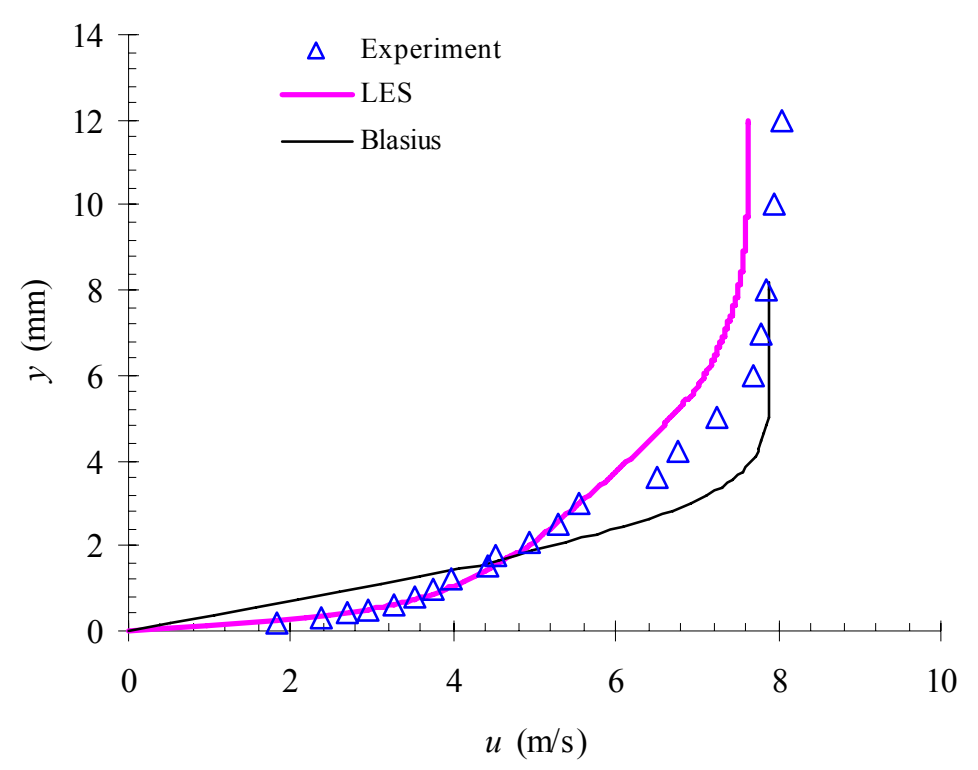

Figure 9 Mean velocity profiles at $x=160 \mathrm{~mm}$, compared with Blasius velocity profile

To identify the flow condition downstream of the reattachment, the mean streamwise velocity profiles at $x=160 \mathrm{~mm}$ are examined. In Fig. 9, the numerical and experimental results of the mean streamwise velocity profiles at $x=160 \mathrm{~mm}$ are compared, and they are also compared with the Blasius profile. It shows a good agreement between the experimental and numerical results for the mean streamwise velocity profiles at this position which is downstream of the 
reattachment. The shape factor $\mathrm{H}_{1}$ values at this position are 1.775 from the experiment and 1.672 from the simulation. Compared with the Blasius velocity profile, the 'fuller' velocity profiles in both experiment and simulation indicate that the flow is turbulent at $x=160 \mathrm{~mm}$. The results shown in Figures 6-9 demonstrate that LES is reasonably capable of capturing the major features in the baseline flow which includes the laminar separation and the transition from laminar to turbulent in a boundary layer at an adverse pressure gradient.

\subsection{Model Verification}

The numerical simulation of the laminar separation bubble is verified by experiment. Results of the mean and fluctuating velocity profiles, the bubble length and the pressure distribution will be compared between the LES simulation and the wind tunnel experiment.

Figure 10 shows the comparison of the mean and fluctuating velocity profiles at $z=0$ in the separation region obtained from the experiment and the numerical simulation, noting that hot wire measurements can not give the sign of the velocity. The mean velocity is the sample mean of the instantaneous streamwise velocity normalized by the velocity of the local potential flow. The fluctuating velocity, $u$ ', was calculated as follows.

$$
u^{\prime}=\sqrt{\frac{\sum_{i=1}^{N}\left(u_{i}-\bar{u}\right)^{2}}{N}}
$$

Where $u_{i}$ is the $i t h$ sample data of the instantaneous streamwise velocity, $\bar{u}$ is the mean of the streamwise velocity, and $N$ is the sample size of one realization.

Both numerical and experimental results in Fig. 10(a) show consistently the inflection points in the velocity profiles at $y$ positions close to the wall in the region of $x=40 \sim 120 \mathrm{~mm}$, indicating a separation region. In the same separation region, the fluctuating velocity shown in Fig. 10(b) is small, further indicating that it is a laminar separation. Both numerical and experimental results 
in Fig. 10 also consistently show that the reattachment occurs at a position between $x=120$ and $x$ $=140 \mathrm{~mm}$, and that the mean velocity profiles are characterized by turbulent boundary layer, at $x$ $=160 \mathrm{~mm}$. The similar variation of the mean and fluctuating velocity profiles positively support the verification of the LES model.

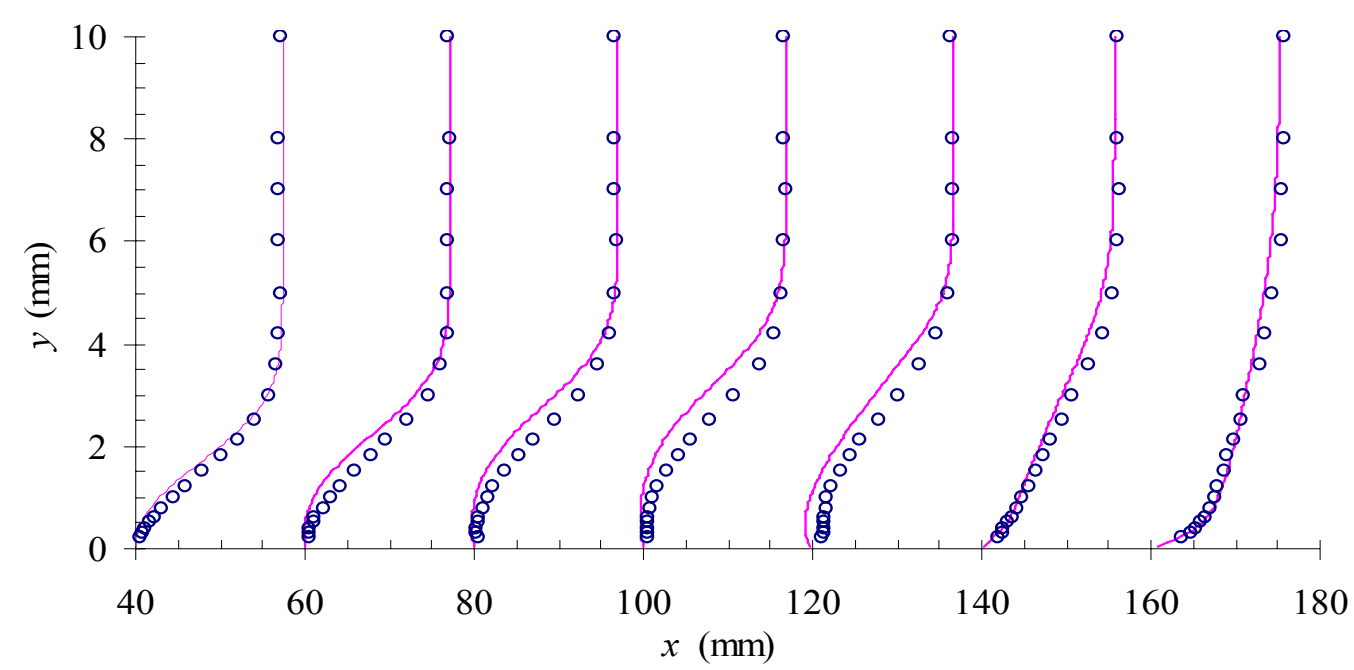

(a)

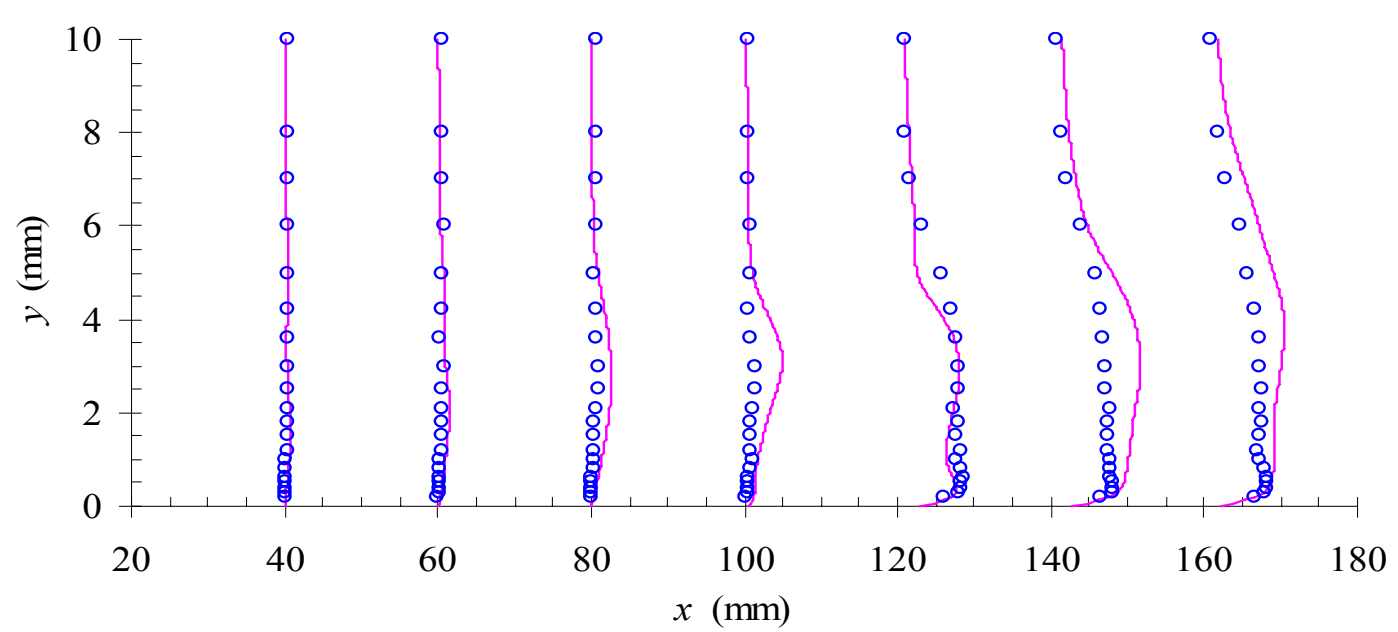

(b)

Figure 10 Velocity profiles in the separation region along the streamwise direction without SJA (a): Mean velocity, $u / U$, (b): Fluctuating velocity $u^{\prime} / U$. -- Numerical simulation, ${ }^{\circ}$ Experiment.

The difference, however, between the numerical and experimental results shown in Fig. 10 is obvious, especially in the transition region of $x=100 \sim 140 \mathrm{~mm}$. The inviscid K-H instability, starting upstream of the position $x=40 \mathrm{~mm}$, develops more quickly in the numerical simulation 
than in the experiment. In the transition from laminar to turbulence, the numerical fluctuating velocity is characterized by stronger development of frictional instability and greater than the experimental one. When the boundary layer flow becomes more steady at $x=160 \mathrm{~mm}$, the difference between the numerical and experimental results is significantly reduced. The difference between the numerical simulation and experiment could be caused by the assumed Blasius velocity profile at the inlet of the computational domain and the freestream turbulence level higher in the numerical simulation than that in the experiment.

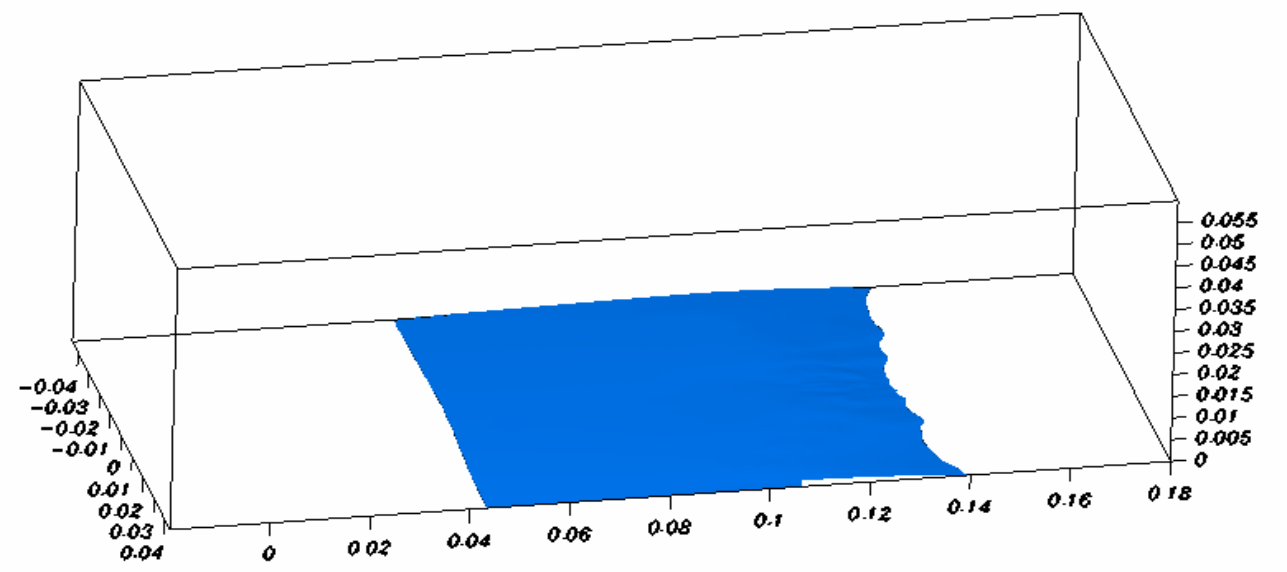

Figure 11 Separation region shown by the zero mean streamwise velocity $u_{\text {avg }}=0$.

(The dimensions are in meter)

The iso-surface of the inflectional points with zero streamwise velocity $u_{\text {avg }}=0$ is used to visualize the shape and dimensions of the separation bubble, as shown in Fig. 11. The minimum and maximum $x$ positions with $u_{\text {avg }}=0$ are used to approximate the separation point and the reattachment points respectively. The separation point on the streamwise centerline occurs at $x=$ $41 \mathrm{~mm}$ and the re-attachment occurs at about $x=134 \mathrm{~mm}$. The corresponding bubble length is $93 \mathrm{~mm}$. Note that the $x$ positions for separation and reattachment points were obtained by averaging respectively the first and the last $x$ positions with $u_{\text {avg }}=0$. As observed in the development of this numerical model, the separation location was well identified at $x=41 \mathrm{~mm}$, as the onset of the separation point consistently occurred at the same $x$ position. However, in the 
breakdown region $(x=120 \mathrm{~mm} \sim 140 \mathrm{~mm})$, the reattachment position was varying from one simulation to another. Consequently, a fixed reattachment point could never be reached. This experience is actually in good agreement with that in [[22]]. Compared with the bubble length which was in the range between 60 and $100 \mathrm{~mm}$ in the wind tunnel tests, the numerical result of the bubble length of $93 \mathrm{~mm}$ is within the range of the experimental result.

Figure 12 compares the experimental and numerical results of pressure distribution in the streamwise direction. As agreed in both experimental measurement and numerical simulation, the pressure distribution is quite 'flat' from $x=40 \mathrm{~mm}$ and $x=110 \mathrm{~mm}$, followed by a quick increase before the pressure gradient is recovered around $x=140 \mathrm{~mm}$. The zone with the 'flat' pressure distribution is where the laminar separation bubble exits. Gaster [[5]] proposed a twoparameter bubble criterion by means of a relationship between the Reynolds number at separation which was based on the momentum thickness $\theta$, and the variation of the free stream velocity over the separation zone. This relation can be described by equation (8).

$$
\bar{P}=\frac{\theta^{2}}{v} \frac{\Delta U}{\Delta x}
$$

Where $\bar{P}$ is pressure parameter, $\Delta U$ is the variation of the free stream velocity over the bubble, and $\Delta x$ is the bubble length. Based on the momentum thickness and the drop of the free stream velocity in the separation region obtained numerically and experimentally, the corresponding $\operatorname{Re}_{\theta}$ and $\bar{P}$ are 300 and -0.14 respectively. In accordance with Gaster's criterion, the separation in the present study is identified as short-bubble separation. This also confirms the height in wallnormal direction of the computational domain, as the short bubble has no influence to the free stream. 


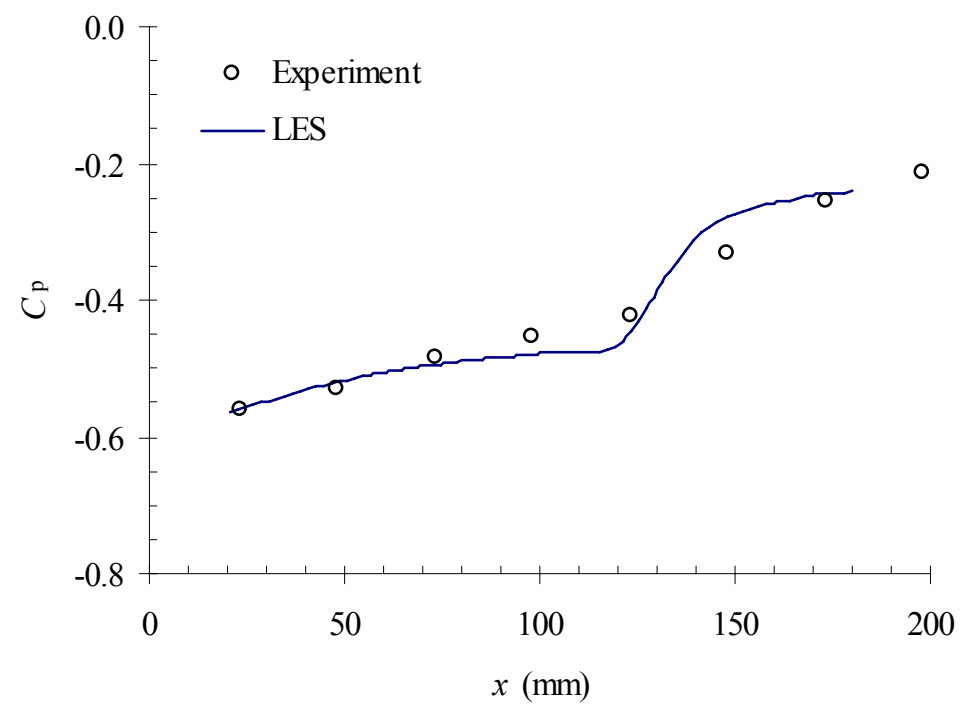

Figure 12 Pressure distributions on the flat plate along the streamwise direction

\subsection{Influence of total time steps}

With a fixed time step, the total number of time steps in a certain time length was numerically tested to decide the minimum number of time steps required for one simulation. If the output parameter, for example the mean velocity profile, does not change significantly with increased total number of time steps, the simulation is regarded converged towards a single solution and the minimum number of time steps should be selected. Figure 13 compares the mean velocity profiles at seven streamwise stations, simulated with total numbers of time steps of 800 and 1600 respectively. It shows that the difference in this major output from simulations with two different total numbers of time steps is insignificant. 


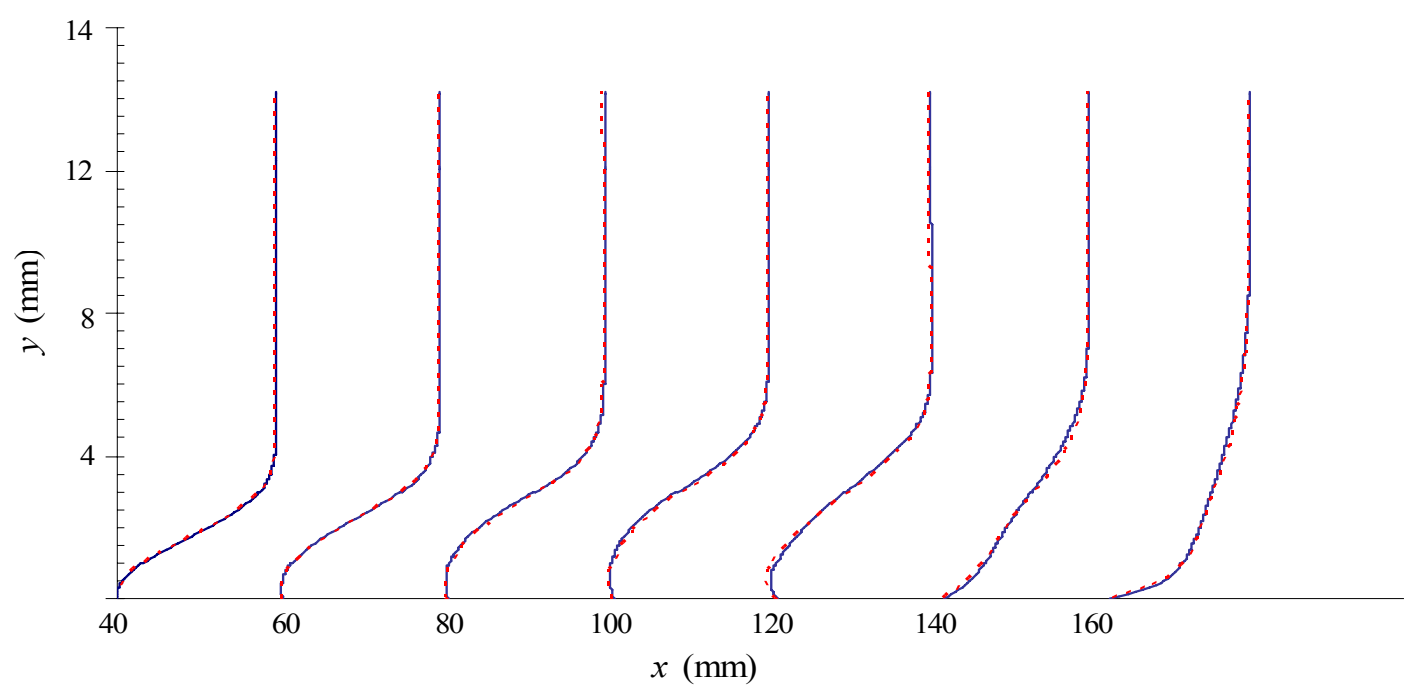

Figure 13 Comparison of mean velocity profiles computed with different time steps. Dashed line: 800 time steps, Solid line: 1600 time steps

However, significant differences exist in other output from the same simulations. Table 2 shows the positions of the separation and reattachment points from two simulations with total time steps of 800 and 1600, compared with the experiment. The separation point appears earlier and the reattachment point does later with 800 time steps than that with 1600 time steps. As a result, the bubble length with 800 time steps is $10 \%$ longer than that with 1600 time steps. The separation bubble with 800 time steps is also outside the range of the bubble length obtained from the experiment. Compared with the experimental results, the positions of separation and reattachment points and the bubble length with 1600 time steps agree well with the experimental results. 1600 time steps were therefore adopted in the simulations.

Table 2 Influence of computational time steps on the separation bubble

\begin{tabular}{lccc}
\hline \hline & $\begin{array}{c}\text { Separation point } \\
x(\mathrm{~mm})\end{array}$ & $\begin{array}{c}\text { Reattachment point } \\
x(\mathrm{~mm})\end{array}$ & $\begin{array}{c}\text { Bubble length } \\
(\mathrm{mm})\end{array}$ \\
\hline 800 time steps & 36 & 138 & 102 \\
\hline 1600 time steps & 41 & 134 & 93 \\
\hline Experiment & $40-60$ & $120-140$ & $60-100$ \\
\hline \hline
\end{tabular}




\section{Simulation with SJA}

In the present numerical simulation with the SJA installed, the cycle involving compression and expansion processes in the cavity of the SJA was not simulated. Instead, the velocity along the centreline of the orifice of the synthetic jet was defined as an inlet to the computational domain at the exit of the SJA. The jet velocity was assumed to be a sine function of time in the $y$ direction, and defined as

$$
\begin{aligned}
& U_{\text {jet }}=0 \\
& V_{\text {jet }}=V_{\text {jet,max }} \sin (2 \pi f t) \\
& W_{\text {jet }}=0
\end{aligned}
$$

Where $U_{j e t}$ and $W_{j e t}$ are the jet velocity values in the streamwise $(x)$ and spanwise $(z)$ directions respectively, and $V_{\text {jet }}$ is the jet velocity along the axial centreline of the orifice in the normal $(y)$ direction. $V_{\mathrm{jet}, \max }$ is the amplitude of the sine function. $f$ is the forcing frequency and $t$ is the time. The first assumption here is that the outlet flow along the axial centreline of the orifice of the SJA is a function of time and a sine wave without consideration of the phase change. It is possible in a real flow that the phase and/or the profile of the jet velocity would vary with time. The second assumption is that the synthetic jet be unaffected by the constriction at the orifice based on our experimental investigation [[8]]. Therefore, the round shape of the orifice of the SJA is replaced by a squared one in the numerical model. The third assumption is that the fluctuating component of the jet velocity be ignored. Figure 2 shows the location of the jet velocity defined as an input in the computational domain. Consistently located as in the experiment, the centreline of the orifice of the SJA in the numerical simulation is on the streamwise centreline of the flat plate and $20 \mathrm{~mm}$ from the inlet of the computational domain. In the 3-D coordinates, the jet velocity was defined at $x=0, y=0$ and $z=0$. 
Based on our experimental results [[9]], the non-dimensional frequency $F^{+}=\frac{f x}{U_{\infty}}$ of the baseline flow was calculated to be in a range of $0.72 \sim 1.41$. In this calculation, the forcing frequency, $f$, was $100 \mathrm{~Hz}$. The length of the laminar separation bubble, $x=60 \sim 100 \mathrm{~mm}$, was taken as the characteristic length and the freestream velocity $U_{\infty}$ was the velocity of the local potential flow. To sufficiently cover the frequency components in the simulation, the time step was set at $\Delta t=0.0002$ second, so that the sine wave with a frequency of $100 \mathrm{~Hz}$ could be numerically sampled by 50 time steps. The simulation of the baseline flow without SJA was run until an equilibrium flow state was reached. The equilibrium flow state then served as the initial conditions for the simulations with the SJA.

\section{Verification of model with SJA}

Figure 14 shows the iso-surface of the vorticity magnitude near the wall when the jet is off and on with a forcing frequency of $100 \mathrm{~Hz}$ and with a maximum jet velocity of $6.0 \mathrm{~m} / \mathrm{s}$. It can be observed from Fig. 14(a) that the iso-surface of the vorticity (vortex layer) separates from the wall when the SJA is switched off. The large-scale waves transfer in streamwise direction and the laminar layer breaks down into streak structure inclining in downstream direction, which was identified in previous work as reported in [[1]]. After the reattachment, the structure becomes finer and more complicated. It demonstrates that LES is sufficiently capable to simulate the transition in a laminar separation boundary layer and the development of a turbulent boundary layer with limited grid points. In comparison with Fig. 14(a), the 'breaking down' when the SJA is switched on, as shown in Fig. 14(b), occurs much earlier (more upstream), and spreading gradually and symmetrically in spanwise direction. The longitudinal vortex structure observed 
under the vortex layer seems to play an important role in accelerating the turbulence to resist the laminar separation when the SJA is switched on.

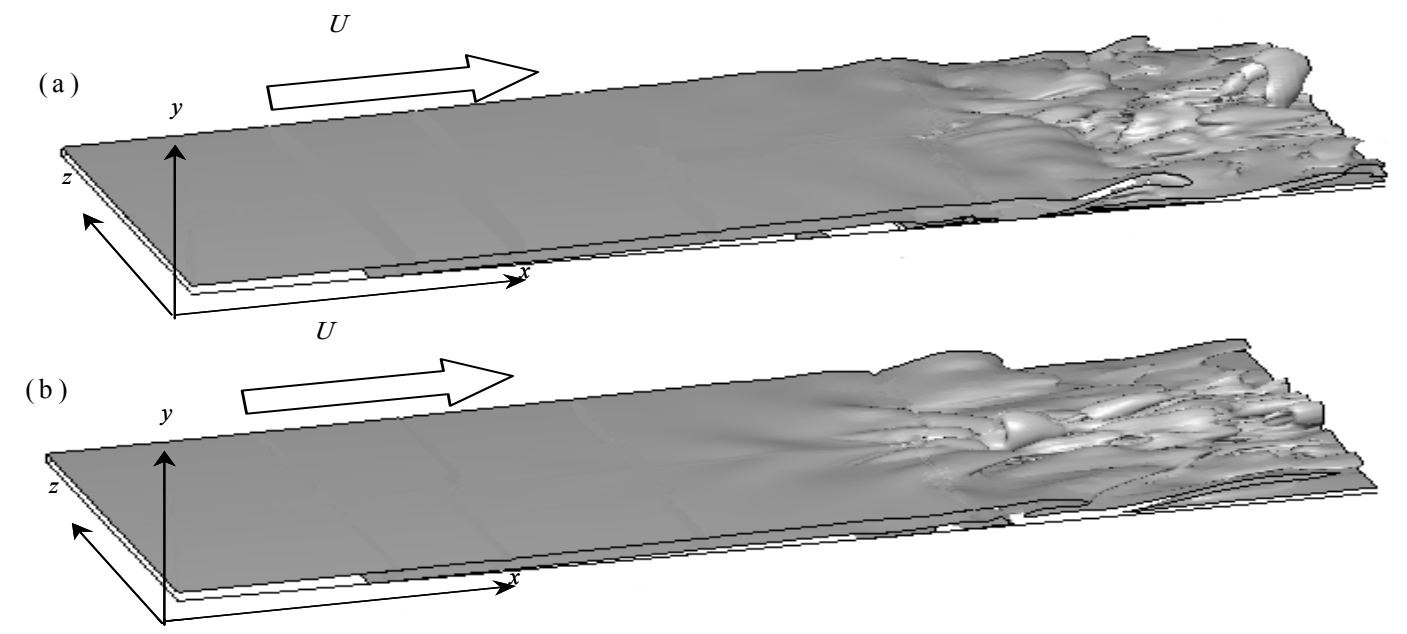

Figure 14 Iso-surface of vorticity near the wall (a) Jet off, (b) Jet on with forcing frequency of $100 \mathrm{~Hz}$ and $V_{\text {jet,max }}=6.0 \mathrm{~m} / \mathrm{s}$.

Experimental results were used to verify the numerical model involving the SJA. Figure 15 shows the comparison of the experimental and numerical results of mean and fluctuating velocity profiles at seven streamwise stations along the streamwise centreline, $z=0$, when the synthetic jet actuator is switched on. The forcing frequency is $100 \mathrm{~Hz}$ and the maximum jet velocity is 6.0 $\mathrm{m} / \mathrm{s}$. Based on the experiments, the separation point in the baseline flow was at a position between $40 \mathrm{~mm}$ to $60 \mathrm{~mm}$, and the reattachment point was between $x=120$ and $x=140 \mathrm{~mm}$ [[9]]. The numerical simulation of the baseline flow consistently predicted that the separation point was at $x=41 \mathrm{~mm}$, and the reattachment point at $x=134 \mathrm{~mm}$, as shown in Figure 10. As shown in Fig. 15(a), both numerical and experimental results of the mean velocity profiles with the inflexion points in the baseline flow are removed by the SJA and the laminar separation bubble does not exist. Figure 15(b) shows that the separation bubble is removed because the fluctuating velocity, triggered by the SJA, enhances the mixing between the shear layer and the rest of the 
boundary layer. As a result, the laminar boundary layer is protected from separation. Considering effective resistance to the laminar separation, the numerical and experimental results agree well.
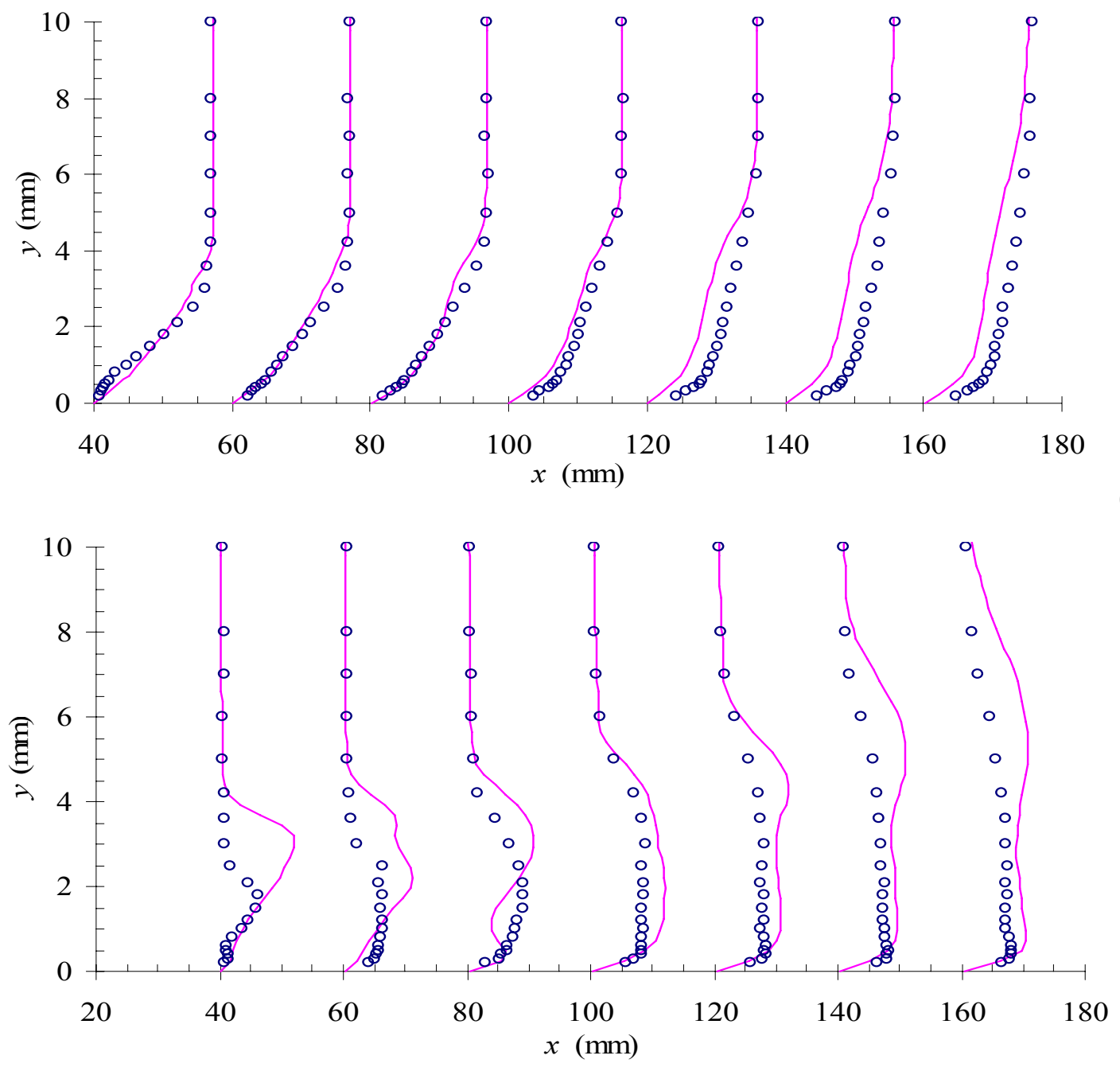

(a)

Figure 15 Comparison of simulated and experimental results when the SJA was on, - simulation, o experiment. (a) mean velocity, (b) fluctuating velocity

The difference between the numerical and experimental results, however, is also obvious as shown in Fig. 15. At $x=40 \mathrm{~mm}$, the inflexion point is still present in the mean velocity profile of the experiment, but not in that of the numerical one. The corresponding fluctuating velocity profiles explain this, as the numerical fluctuating velocity is greater, especially at $y=2.0 \sim 4.0$ $\mathrm{mm}$, than the experimental one. This shows that the synthetic jet in the numerical model accelerated the transition from laminar to turbulence more quickly than the synthetic jet did in 
the experiment. The differences between the numerical and experimental results of both mean and fluctuating velocity profiles are increasing downstream. At the positions close to the wall, $y$ $<1.0 \mathrm{~mm}$, the numerical fluctuating velocity agrees relatively well with the experimental one, although the differences between the numerical and experimental results of mean velocity profiles at $x=100 \sim 160 \mathrm{~mm}$ are noticeable. As we experienced, the baseline flow numerically simulated is very sensitive to the disturbance triggered by the SJA. The major difference between the numerical and experimental results is shown by the fluctuating velocity profiles in the upper region of the boundary layer. Starting from $x=120 \mathrm{~mm}$, as shown in Fig. 15(b), an 'overhang' on the fluctuating velocity profile is growing and moving towards the edge of the boundary layer downstream. This 'overhang' may be due to the energy accumulation in the simulation. Further increasing the width of the computational domain and adding a buffer box at the outlet end of the computational domain may reduce the 'overhang'.

Figure 16 shows the comparison of boundary layer thickness for the results shown in Fig. 15. As shown in Figure 15, the numerical and experimental mean velocity profiles agree better in the region from $x=40 \mathrm{~mm}$ to $x=80 \mathrm{~mm}$ than that in the rest of the region. However, the comparison of the boundary layer thickness in Figure 16 shows more difference between the numerical and experimental results in the region from $x=40 \mathrm{~mm}$ to $x=80 \mathrm{~mm}$ than that in the rest of the region. In the region from $x=100 \mathrm{~mm}$ and $x=160 \mathrm{~mm}$, the numerical boundary layer thickness agrees well with the experimental one, while the comparison in the same region in Figure 15 shows less agreed results between the simulation and the experiment.

As described previously, Blasius velocity profile was assumed at the inlet of the computational domain with an adjusted freestream velocity. Instead of simulating the physical cycle in the actuator, a function of the jet velocity at the center of the exit of the SJA was 
specified. On the other hand, as we have experienced, the flow simulated was very sensitive to the disturbance triggered by the synthetic jet. The width of the computational domain and the grid ratio may also require further improvement when more computational resources are provided. Therefore, the assumptions made in the initial and boundary conditions and some of the numerical methods adopted could all contribute to the errors in the numerical simulation.

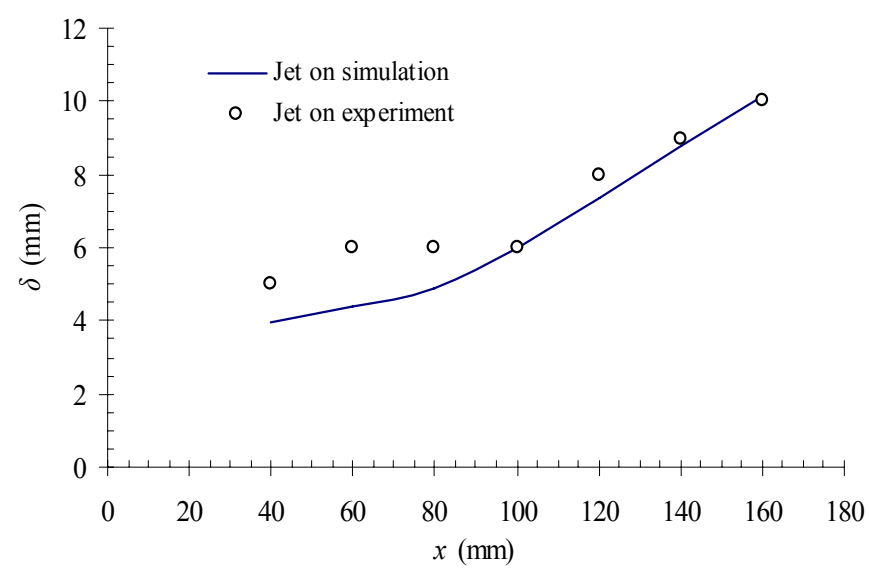

Figure 16 Comparison of numerical and experimental results of boundary layer thickness along the streamwise centerline

In the wind tunnel experiment, it was noticed that the fluctuating velocity with the SJA operating at a forcing frequency of $100 \mathrm{~Hz}$ and forcing amplitude of $\pm 7.5 \mathrm{~V}$ was smaller than that without the synthetic jet at $x=160 \mathrm{~mm}$ [[9]]. This has led to an idea of enabling the SJA to play dual roles in enhancing as well as reducing the turbulence to meet various control objectives [9]. Figure 17 compares the fluctuating velocity profiles without the jet to that with the jet at a forcing frequency of $100 \mathrm{~Hz}$ for both experiment and simulation, at $x=160 \mathrm{~mm}$. It shows that the numerically simulated fluctuating velocity with the jet on is also smaller than that without the jet. This observation from the numerical simulation is consistent with that from the experiment. 


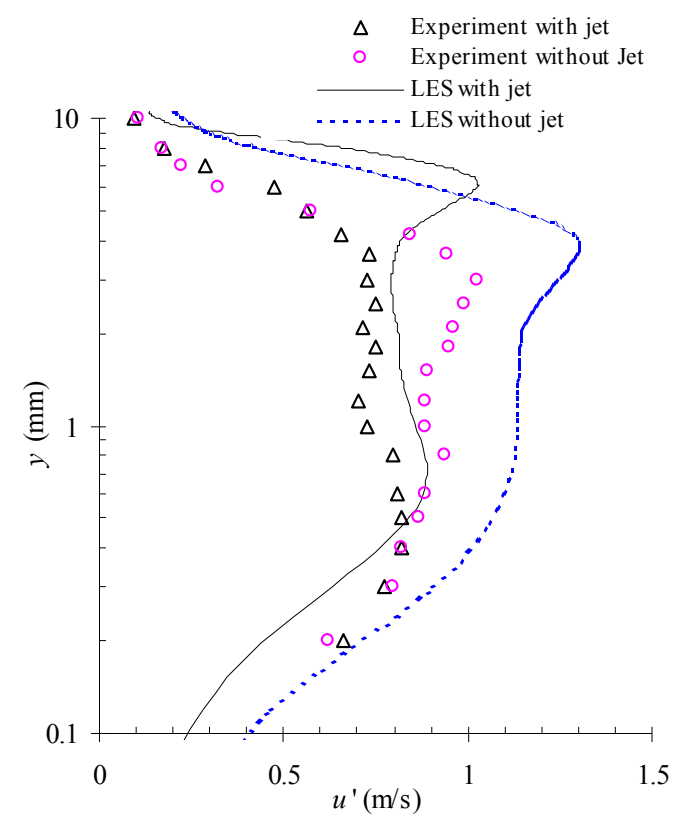

Figure 17 Comparison of fluctuating velocity profiles at $x=160 \mathrm{~mm}$

\section{Interaction between the synthetic jet and the baseline flow}

In the wind tunnel experiment, the traverse of the hotwire probe was restricted to streamwise and normal directions. The experimental data only provided information of the streamwise velocity measured at a certain number of $(x, y)$ positions along the streamwise centrerline. To extend our knowledge about the interaction between the synthetic jet and the baseline flow, the verified numerical model was used.

Figure 18 shows the iso-surfaces of the instantaneous vorticity (a) and the averaged positions with zero streamwise velocity $u_{\text {avg }}=0(\mathrm{~b})$, projected on $x-z$ plane. The instantaneous vorticity of 5600 in Fig. 18(a) was chosen because it was a new vorticity value introduced by the synthetic jet. Next to the side walls of the computational domain, the behaviour of the flow with the synthetic jet is similar to that without the synthetic jet, as the flows in both cases break down in thin streaks around $x=100 \sim 120 \mathrm{~mm}$. (Note that representation of the instantaneous vorticity next to the side walls may be biased due to the coarser grids). The vortices introduced by the synthetic 
jet propagate in both streamwise and spanwise directions. However, development of the vorticity introduced by the synthetic jet remains in the region symmetric about the streamwise centreline and does not reach the sidewalls of the computational domain. The limited region of this vorticity development may indicate that to make a SJA effective in both streamwise and spanwise directions, certain distance between the SJA and the separation bubble is required.

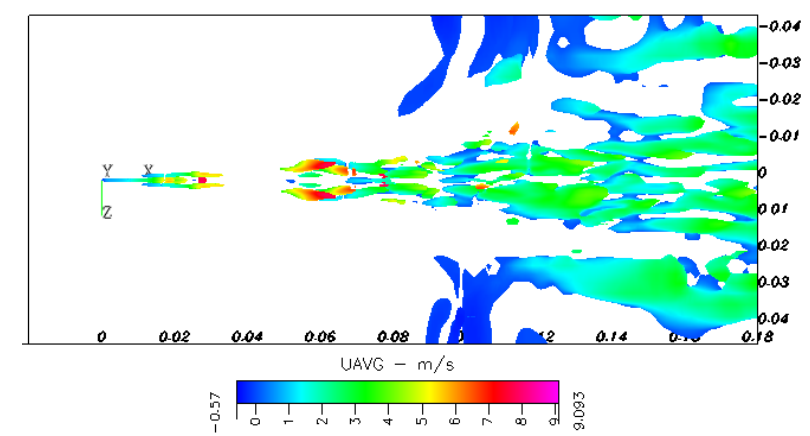

(a)

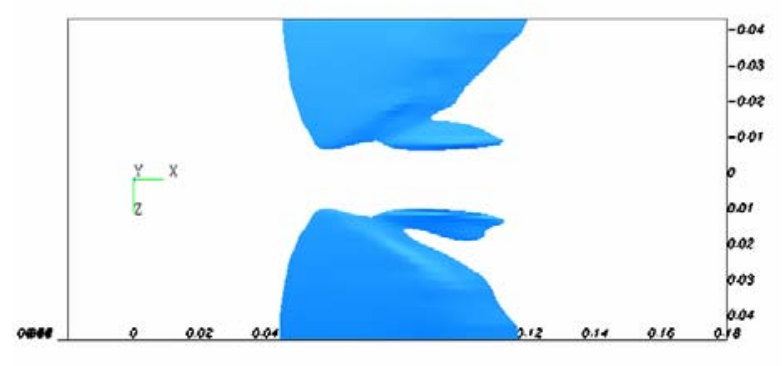

(b)

Figure 18 Top view of the iso-surface of instantaneous vorticity $=5600$ (a) and the iso-surface of time-averaged streamwise velocity $=0$ (b)

The vorticity concentrated on both sides of the streamwise centreline is further confirmed by the iso-surface of the averaged positions with $u_{\text {avg }}=0$, in Fig. 18(b). As shown by the region with $u_{\text {avg }}=0$ removed in Fig. 18(b), the laminar separation originally in the baseline flow is successfully resisted by the synthetic jet. Although the bubble is not entirely removed, the laminar separation region symmetric about the streamwise centreline has disappeared. The minimum width of the eliminated separation bubble is about $16 \mathrm{~mm}$ as measured at $x=90 \mathrm{~mm}$, equivalent to 32 times the jet orifice diameter. Such information should be necessary for determining the distance between two SJAs in spanwise direction.

The developed model will be applied to help us understand the physics involved in the experiments. For example, the experimental results showed that the effect of the forcing frequency on the effect of flow control was more significant than that of the forcing amplitude 
$[8,9]$. The numerical simulation may provide more detailed information for us to understand why. In Figure 19 are two sample results of the simulation, the spanwise vortices in the $x-y$ plane at $z=0$ in (a) and the streamwise vortices which are a pair of vortices on the $y-z$ plane at $x=17$ $\mathrm{mm}$. The spanwise vortices at different $z$ positions and the streamwise vortices at different $x$ positions will be used to analyze the physics associated with the synthetic jets.
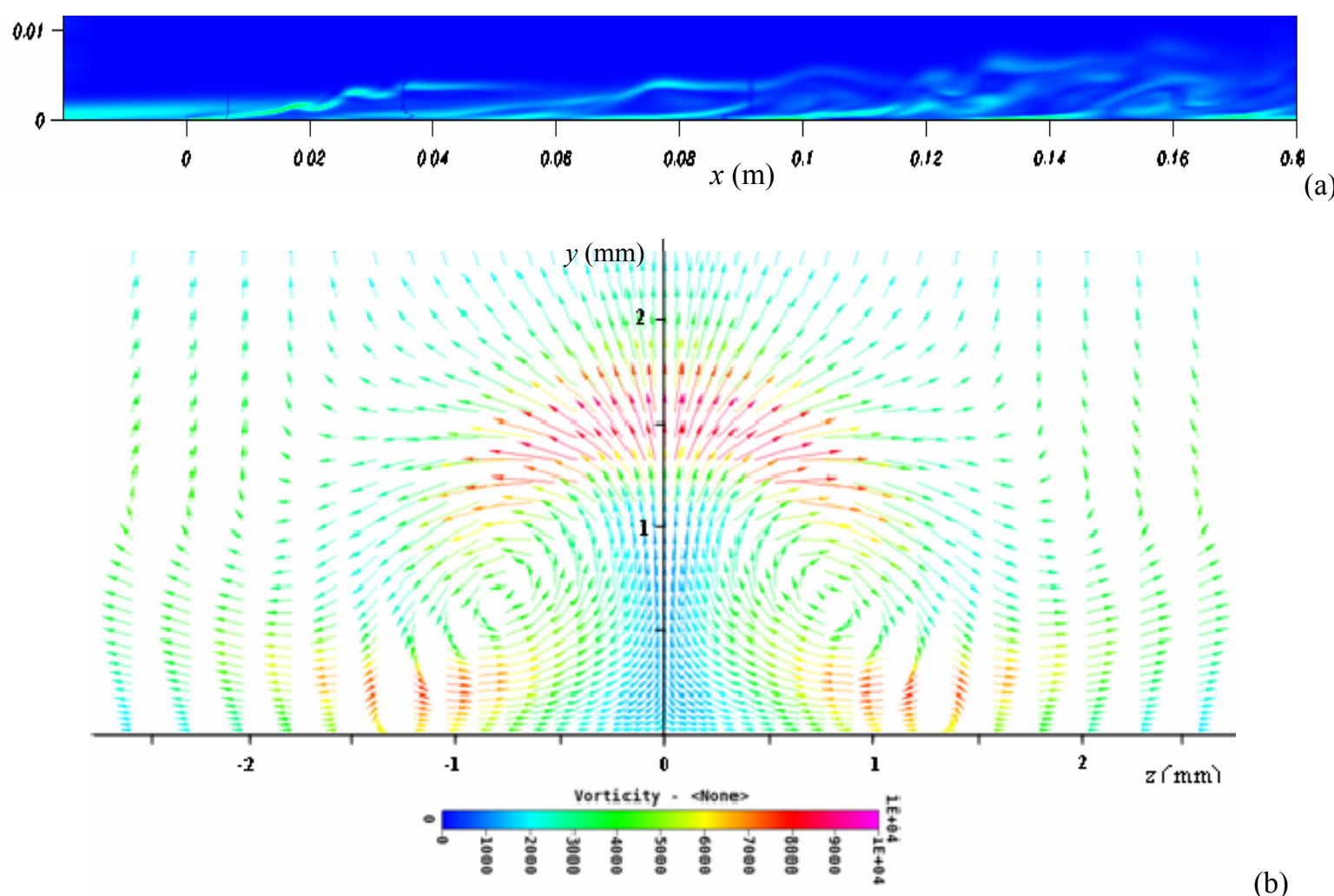

(a)

Figure 19 a) Spanwise vortices at $z=0$, b) Streamwise vortices at $x=17 \mathrm{~mm}$, Forcing frequency $=100 \mathrm{~Hz}$

\section{Conclusion}

A numerical model of a baseline flow was prepared for investigating the SJA in flow separation control. It simulates the boundary layer flow field enclosing a 'short' laminar separation bubble caused by adverse pressure gradient. A commercial code was used to solve the governing equations. The computational domain was three-dimensional and covered the exit of the SJA. LES was employed to achieve the optimal balance between the computational resources 
and the accuracy of the numerical modelling. Dynamic Smagorinsky model was used for the sub-grid scale stress (SGS) terms. The initial and boundary conditions were defined using or referring to our wind tunnel experiments.

The simulated separation bubble was visualized in various ways to identify the laminar-short separation bubble. The averaged position with $u_{a v g}=0$ in the inflectional velocity profile was used to identify the edge of the bubble, including the separation point and the reattachment point. The iso-surface of vorticity was used to show the quick transition from laminar to turbulence, characterised in a 'short' laminar separation bubble and the mixing of the flow after the transition. The exponential variation of the maximum fluctuating velocity along the streamwise direction indicated the linear instability. The influence of the number of time steps was investigated.

The model for the baseline flow was verified by comparing the numerical and experimental results including the mean and fluctuating velocity profiles at seven stations on the streamwise centerline in the separation zone. The agreement between the simulation and the experiment is reasonably good. The identified separation bubble and the model verification demonstrated that LES was reasonably capable for capturing the basic features of a 'short' separation bubble, including the transition from laminar to turbulent. However, we must bear in mind that the difficulty in simulating the transition from laminar to turbulent is still unavoidable.

Based on a reasonable agreement between the experimental and numerical results in the separation zone of the baseline flow, a synthetic jet was inserted at a position upstream of the laminar separation bubble in the LES model for the baseline flow. The simulated mean and fluctuating velocity profiles with the synthetic jets were compared with the experimental ones at seven positions on the streamwise centerline. In terms of the actuation of the SJA, the 
experimental and numerical results agreed well as both showed effective elimination of the separation bubble when the forcing frequency was $100 \mathrm{~Hz}$ and the maximum jet velocity at the exit of the SJA was $6.0 \mathrm{~m} / \mathrm{s}$.

The iso-surface of inflectional points with $u_{a v g}=0$ was used to visualize the separation zone eliminated by the SJA, and the iso-surface of vorticity was used to help understand the associated physics. They were used to show the interaction between the synthetic jet and the flow to be controlled.

As we experienced, the baseline flow was very sensitive to the disturbance triggered by the SJA. The level of difficulties in simulating the transition from laminar to turbulence and in handling the diffusion increased when the SJA was switched on. However, numerical simulation has shown its potential in helping the development of SJAs. The outcomes of numerical simulations should contribute to shortening the time on realizing the use of SJAs in a real world.

\section{Acknowledgments}

Assistance provided by Mr. Peter Brady in CFD is gratefully appreciated.

\section{References}

[1] Alam, M. and Sandham, N. D., Direct Numerical Simulation of 'Short' Laminar Separation Bubbles with Turbulent Reattachment, J. Fluid Mech, 2000, Vol. 403, pp. 223-250.

[2] Allan, B. G., Holt, M., and Packard, A., Simulation of a controlled airfoil with jets, NASA/CR-201750, ICASE Report No. 97-55, October 1977.

[3] Gad-el-Hak, M., Flow Control: Passive, Active and Reactive Flow Management (section 8.10.2), Cambridge University Press, Cambridge, 2000. 
[4] Galperin, B. and Orzag, S. A., Eds., Large Eddy Simulation of Complex Engineering and Geophysical Flows, Cambridge University Press, Cambridge, 1993.

[5] Gaster, M., The Structure and Behaviour of Laminar Separation Bubbles, A.R.C. R\&M Report No. 3595, 1969.

[6] Glezer, A. and Amitay, M., Synthetic jets, Annu. Rev. Fluid Mech., 2002, 34:503-29.

[7] Hatman, A. and Wang, T., A Prediction Model for Separated-Flow Transition, ASME paper, GT-29-237, ASME Turbo Expo ‘98’, Stockholm, Sweden, 1998.

[8] Hong, G., Effectiveness of micro synthetic jet actuator enhanced by flow instability on controlling laminar separation caused by adverse pressure gradient, Sensors \& Actuators, Physics A, Vol. 132, Issue 2, pp607-615, 2006.

[9] Hong, G., Lee, C., Ha, Q.P., Mack, A.N.F. and Mallinson, S. G., Effectiveness of synthetic jets enhanced by instability of Tollmien-Schlichting waves, AIAA paper, 2002-2832, 2002.

[10] Honohan, A. M., Amitay, M. and Glezer, A., Aerodynamic control using synthetic jets, AIAA paper, 2000-2401, 2000.

[11] Kral, L. D., J.F. Donovan, J. F., Cain, A. B. and Cary, A. W., Numerical simulation of synthetic jet actuator, AIAA paper $97-1824,28^{\text {th }}$ AIAA Fluid Dynamics Conference, Snowmass Village, CO, June 29 - July 2, 1997.

[12] Li, Y. and Ming, X., Control of two dimensional jets using miniature zero mass flux jets, Chinese Journal of Aeronautics. August 2000, Vol. 13, No. 3, pp. 129-133.

[13] Lilly, D. K., A Proposed Modification of the Germano Subgrid Scale Closure Method, Physics of Fluids, 1992, Vol 4, pp 633-634. 
[14] Lockerby, D. A. and Carpenter, P. W. Modeling and design of microjet actuation, AIAA Journal, February 2004, Vol. 42, No. 2, pp220-227.

[15] Ming, X., Dai, C. and Shi, S., A new phenomenon of acoustic streaming, ACTA MECHANICA AINICA, August 1991, Vol. 7, No. 3, 193-198.

[16] Mittal, R. and Rampunggoon, P., On the virtual aeroshaping effect of synthetic jets, Brief Communications, Physics of Fluids, April 2002, Vol. 14, No. 4, pp 1533-1536.

[17] Mittal, R., Rampunggoon, P. and Udaykumar, H. S., Interaction of a synthetic jet with a flat plate boundary layer, AIAA paper, 2001-2773, 2001.

[18] Na, Y. and Moin, P. Direct Numerical Simulation of a Separated Turbulent Boundary Layer, J. Fluid Mech, 1998, Vol. 374, pp. 379-405.

[19] Ozawa, T., Hong, G. and Mack, A. N. F., CFD modellng of synthetic jets in a boundary layer at adverse pressure gradient, Proceedings of 5th Pacific Symposium on Flow Visualisation and Image Processing (5PSFVIP), Daydream Island, Australia, 27-29th September 2005 .

[20] Parekh, D., Palaniswamy, S. and Goldberg, U., Numerical simulation of separation control via synthetic jets, AIAA paper, 2002-3167, 2002.

[21] Ravi, B. R., Mittal, R. and Najjar, F. M., Study of three-dimensional synthetic jet flow fields using direct numerical simulation, 42nd AIAA Aerospace Sciences Meeting and Exhibit, Reno, NV, 5-8 January, 2004.

[22] Redford, J. A. and Johnson, M. W., Predicting transitional separation bubbles, Proc. TURBOEXPO 2004, International Gas Turbine Congress, Austria, 14-17 June 2004. 
[23] Rist, U., Instability and transition mechanisms in laminar separation bubbles, VKI/RTO-LS Low Reynolds Number Aerodynamics on Aircraft including Applications in Emergency UAV Technology, Rhoda-Saint-Gense, Belgium, 24-28 November 2003.

[24] Rist, U., On instabilities and transition in laminar separation bubbles, Proc. CEAS Aerospace Aerodynamics Research Conference, Cambridge, UK, 10-12, June 2002.

[25] Rizzetta, D. P., Visbal, M. R. and Stanek, M. J., Numerical investigation of synthetic jet flowfields, AIAA paper, 98-2910, 29 th $^{\text {th }}$ AIAA Fluid Dynamics Conference, Albuquerque, NM, June 15-18, 1998.

[26] Rumsey, C.L., "Successes and challenges for flow control simulations", AIAA-2008$4313,2008$.

[27] Sagaut, P., Large Eddy Simulation for Incompressible flow, second edition, Springer, 2004.

[28] Seifert, A., Theofilis, V. and Joslin, R. D., Issues in active flow control: theory, simulation and experiment, AIAA paper, 2002-3277, 2002.

[29] Smagorinsky, J., General Circulation Experiments with the Primitive Equations, I. The Basic Experiment, Monthly Weather Review, Vol. 91, 1963, pp 99-96.

[30] Smith, B. L. and Glezer, A., Vectoring and small-scale motions effected in free shear flows using synthetic jet actuators, AIAA paper, 97-0213, 1997.

[31] Wilson, P. G. and Pauley, L. L., Two-dimensional large eddy simulation of a transitional separation bubble. Symp. On Separated and Complex Flows, ASME/JSME Fluid Engineering Conf., 1995. 
[32] Wissink, J. G. and Rodi, W., DNS of Transition in a Laminar Separation Bubble, In I. P. Castro and P.E. Hancock, editors, Advances in Turbulence IX, proceedings of the Ninth European Turbulence Conference, CIMNE, 2002. 